\title{
The Attraction and Compromise Effects in Bargaining: Experimental Evidence
}

\author{
Fabio Galeotti* Maria Montero ${ }^{\dagger}$ Anders Poulsen ${ }^{\ddagger}$
}

February 16, 2021

\begin{abstract}
We experimentally investigate, in an unstructured bargaining environment with commonly known money payoffs, the Attraction Effect and Compromise Effect (AE and CE) in bargaining, namely a tendency for bargainers to agree to an intermediate option (CE), or to an option that dominates another option (AE). We conjecture that the relevance of the $\mathrm{AE}$ and $\mathrm{CE}$ in bargaining is constrained by how focal the feasible agreements' payoffs are. We indeed observe that there are significant AEs and CEs, but these effects are mediated by the efficiency and equality properties of the feasible agreements. Due to the allure of equality, the effects are harder to observe when an equal earnings contract is available. Decoys are more effective in shifting agreements from a very unequal contract to a less unequal one rather than the reverse. Keywords: bargaining; attraction effect; compromise effect; focality; equality; efficiency.
\end{abstract}

JEL Classification: C70; C72; C92.

\section{Introduction}

The motivation for this paper comes from two behavioral regularities, found in individual decision making studies, namely the Attraction Effect (AE) and the Compromise Effect (CE) (see Huber et al., 1982; Huber and Puto, 1983; Simonson, 1989). Suppose a person must choose between say two apartments, $A$ and $B$, that differ in two salient attributes, such as size and location.

\footnotetext{
${ }^{*}$ Univ Lyon, CNRS, GATE UMR 5824, F-69130 Ecully, France.

${ }^{\dagger}$ School of Economics, University of Nottingham, Nottingham NG7 2RD, UK.

‡Corresponding author. School of Economics and CBESS, University of East Anglia, Norwich NR4 7TJ, UK. We thank Kevin Grubiak for lab assistance. Thanks also to Arnold Polanski, Neil Stewart, Mich Tvede, and seminar participants at Aarhus, CBESS, GATE, Vienna, the 2016 FUR meeting, the 2016 International Conference of the French Association of Experimental Economics, the 2016 NIBS meeting at Warwick Business School, and the 2017 GATE-Lab Workshop on "Social and Moral Norms" for very helpful comments. All errors are ours. This research was funded by British Academy/Leverhulme Small Grant SG132438. Poulsen acknowledges support from the ESRC Network for Integrated Behavioural Science (NIBS) (grant ES/K002201/1). Galeotti acknowledges financial support of IDEXLYON from Universit de Lyon (INDEPTH, IDEX-SBP-2018-03) within the Programme Investissements dAvenir (ANR16-IDEX-0005). This research was performed within the framework of the LABEX CORTEX (ANR-11-LABX-0042) of University of Lyon, within the program Investissements d'Avenir (ANR-11-IDEX-007) operated by the French National Research Agency (ANR).
} 
Two such apartments are shown in Figure 1 (where higher values of both size and location are assumed to be more desirable). The choice is non-trivial since $A$ is smaller but better located than $B$. Suppose a third apartment, $C$, which is dominated by say apartment $B$ but not by $A$, is added to the choice set (see Figure 1a). ${ }^{1}$ The Attraction Effect (AE) arises when the decision maker is more likely to choose $B$ when the set of alternatives is $\{A, B, C\}$ than $\{A, B\}$. This violates the axiom known as Regularity (see Luce, 1977), which states that the probability of choosing an option cannot increase when the choice set is expanded.

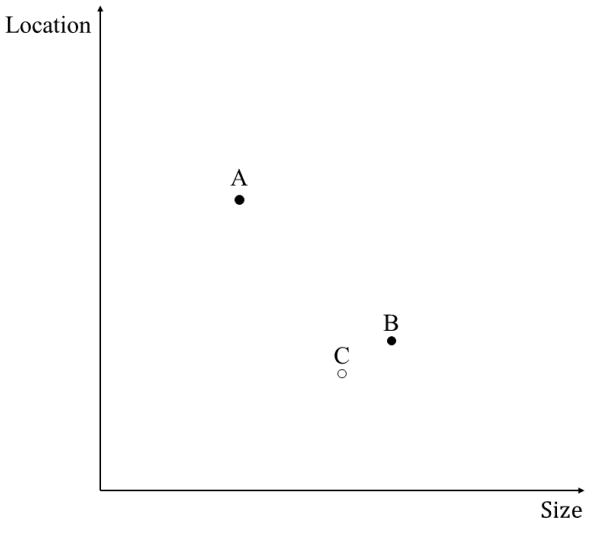

(a) Attraction Effect.

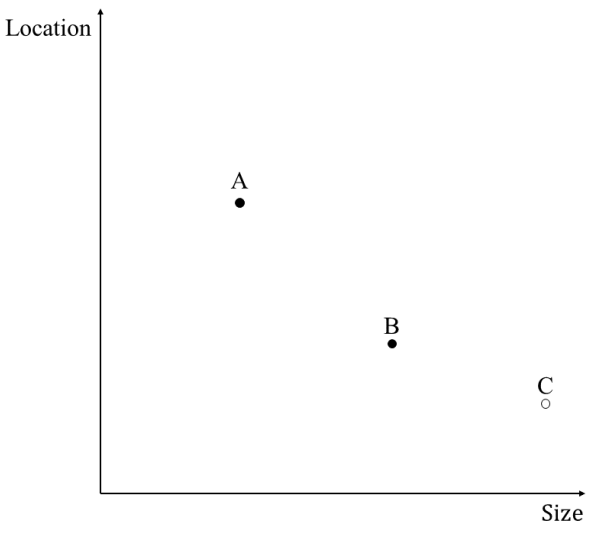

(b) Compromise Effect.

Figure 1: Attraction and Compromise Effects in individual choice.

Consider then the case where adding the third alternative $C$ makes option $B$ a compromise (second best on each attribute dimension), as shown in Figure 1b. The Compromise Effect (CE) occurs when the decision maker is more likely to choose $B$ when the set of options is $\{A, B, C\}$ than when it is $\{A, B\}$, once more a violation of regularity.

The $\mathrm{AE}$ and $\mathrm{CE}$ have been found to significantly influence choice in a variety of situations, such as product choice (Doyle et al., 1999; Munro and Popov, 2013; Milberg et al., 2014), contingent valuation (Bateman et al., 2008), job candidate selection (Highhouse, 1996; Slaughter et al., 2006; Kuncel and Dahlke, 2020), sampling decisions (Trueblood et al., 2013; Noguchi and Stewart, 2014), elections (Pan et al., 1995; Herne, 1997), choice among gambles (Wedell, 1991; Herne, 1999; Beauchamp et al., 2019; de Haan and van Veldhuizen, 2015), legal judgments (Kelman et al., 1996), menu choices in a restaurant (Pinger et al., 2016), and partner choice (Sedikides et al., 1999). Various explanations for these effects have been put forward (see Section 2 below).

As far as we are aware, all the existing empirical research on the AE and CE has (with only very few exceptions, described in the next section) been concerned with individual choice situations. In this paper we ask if there are also AEs and CEs in interactive settings, namely bargaining situations, where two players can collaborate in a number of mutually beneficial ways, but there is a conflict about exactly what form the collaboration should take. Negotiation researchers as well as practitioners should be interested in learning the conditions under which adding a dominated

\footnotetext{
${ }^{1} C$ is dominated by $B$ in the sense that $B$ is strictly better than $C$ on both attribute dimensions.
} 
option to an existing menu of options is likely to affect the bargaining outcome, and when one can increase the likelihood of an agreement on a certain 'target' option by manipulating the menu of feasible agreements such that the target becomes a compromise.

To see the managerial relevance, consider a hiring committee whose members must agree on what candidate to select from a small number of job applicants. There may be only a few (two or three) applicants, or prior shortlisting has reduced the set to such a small number. Another managerial example is intra-organizational negotiation, where a committee consisting of two department heads (of say production and advertising) must agree to a product design and advertising policy. Due to various technological constraints, or to limits on how many options the committee want or can consider, there is only a small number of feasible policies. A third example is collective bargaining between employers and unions, where the negotiation focuses on which wage-working conditions package to agree on. In these situations it is often the case that there are few options, some of which may be compromises, others may be dominated, and so it becomes natural to ask if there exist AEs and CEs. ${ }^{2}$

We study the $\mathrm{AE}$ and $\mathrm{CE}$ in bargaining by letting players negotiate over a set of options (referred to as "contracts"), where each contract has two attributes, namely how much money each player gets if they agree to the contract in question. ${ }^{3}$ See Figure 2 below. The set of feasible contracts (the contract set) either consists of two, $S=\{A, B\}$, or three contracts, $T=\{A, B, C\}$. An agreement is an element from the contract set (the players cannot agree to more than one element, or to a lottery over contracts). If they agree to say contract $B$, then Player 1 (2) gets a monetary payoff, $B_{1}\left(B_{2}\right)$. If they fail to agree, each bargainer gets zero.

Each axis in Figure 2 measures each player's subjective payoff. In our experiment we assume that players are self-interested and risk neutral, such that the utility from a contract equals the money it pays out. Of course, it is possible that players have social preferences (see, e.g., Fehr and Schmidt, 1999; Bolton and Ockenfels, 2000; Charness and Rabin, 2002). In this case the ordinal relationships between the contracts in money space could differ from the ones in utility space. Nevertheless, we show, in the Online Appendix, that the money based rankings are robust to (moderate) other-regarding preferences. More precisely, our findings for the AE and CE also hold for inequity averse bargainers, as long as the concerns for disadvantageous and advantageous inequality $(\alpha$ and $\beta$ ) are not too large. Similarly, our results are robust to both maximin and efficiency

\footnotetext{
${ }^{2}$ Other, non-managerial, examples include a couple who are about to buy a new car, or parents deciding which school to send their child to.

${ }^{3}$ The fact that the objects of choice specify a distribution of money across a group of people clearly distinguishes bargaining from the existing individual choice research on $\mathrm{AE}$ and $\mathrm{CE}$, where individuals choose between (hypothetical descriptions of) real goods that differ in various physical attributes. Another difference, pointed out by a referee, is that in individual multi-attribute choice the individual cares about each attribute, while in our bargaining set-up a purely self-interested person cares only about one attribute, namely his own money payoff - but players can clearly not freely choose their preferred contract since the other person can veto it. We believe that restricting attention to bargaining over pairs of money payoffs is a reasonable simplification. It also provides a direct link to bargaining theory. We think that the presence of exact monetary values placed by each player on the alternatives makes context effects harder to observe because it introduces equality and total payoff considerations. In the absence of these considerations, we would expect the effects to be even stronger. Future research can study more complex settings where players bargain over goods described by multiple attributes.
} 
preferences as long as the concerns for these properties are not too strong.

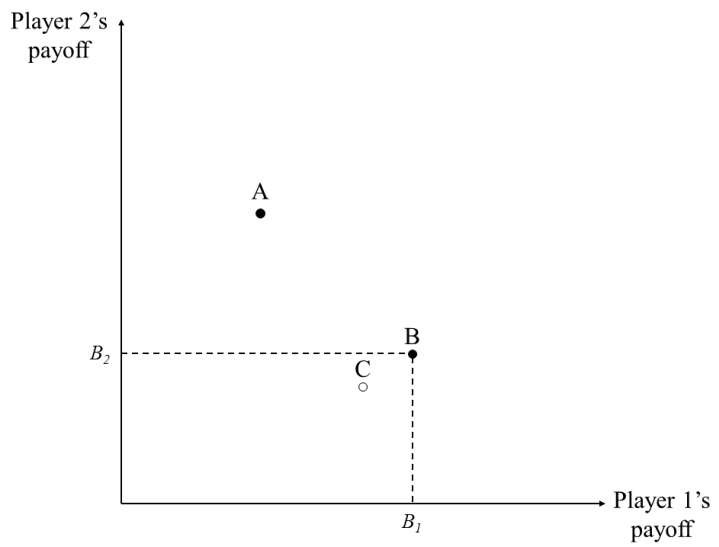

(a) Attraction Effect.

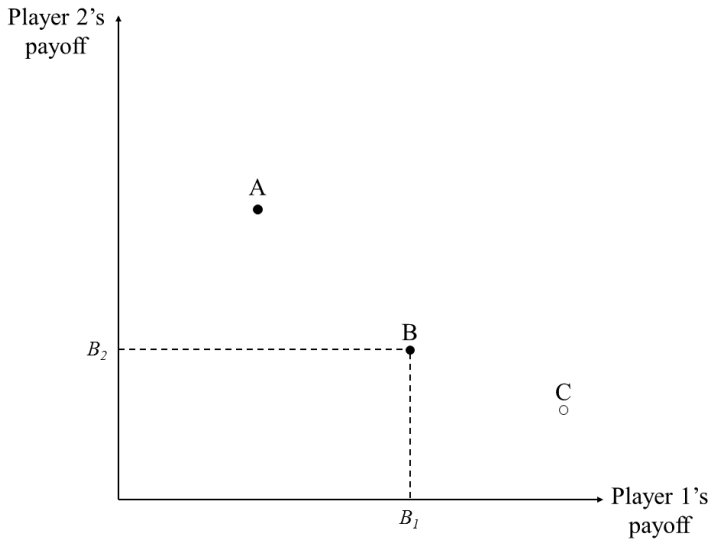

(b) Compromise Effect.

Figure 2: The Attraction and Compromise Effects in bargaining.

In Figure $2 \mathrm{a}$ the contract $C$ is strictly dominated by $B$, but not by $A$. We define the $\mathrm{AE}$ in bargaining as follows: the bargainers are more likely to agree on $B$ when the contract set is $T=$ $\{A, B, C\}$ than when it is $S=\{A, B\}$. In terms of the data, the AE means that the proportion of agreements on $B$ is higher in the first than the second case. In Figure $2 b$, adding contract $C$ makes $B$ a compromise (i.e., each player's second best contract in terms of money payouts). The $\mathrm{CE}$ in bargaining arises when the bargainers are more likely to agree on $B$ when the contract set is $\{A, B, C\}$ than when it is $\{A, B\}$. As we explain below, we regard both the $\mathrm{AE}$ and the $\mathrm{CE}$ as a violation, at the level of the aggregate data, of the Independence of Irrelevant Alternatives condition, IIA (Nash, 1950), which states that if the bargainers agree to some option when there is a large set of alternative options available, then the same option is agreed to when the set of alternatives is reduced. ${ }^{4}$

Why might we expect there (not) to be an AE and CE in bargaining in the first place? Consider first cooperative bargaining theory (see Thomson, 1994). As we show in Section 5 below, both the Nash and the Kalai-Smorodinsky Bargaining Solution (Nash, 1950; Kalai and Smorodinsky, 1975) rule out the AE, and they yield different predictions regarding the CE. ${ }^{5}$ Among ordinal bargaining solutions, the Fallback Solution (Brams and Kilgour 2001; see also de Clippel and Eliaz 2012) predicts both the AE and CE, while the Ordinal Egalitarian Solution (Conley and Wilkie, 2012), predicts the CE but rules out an AE.

A well-known hypothesis for coordination and bargaining situations (see Schelling, 1960; Myerson, 1989) is that the bargainers can overcome the coordination problem by identifying a focal

\footnotetext{
${ }^{4}$ As already remarked earlier, it is possible that players have preferences such that the location of contracts in utility space differ from the one in monetary reward space; what then appears to be an AE (CE) might be a different context effect. However, regardless of whether it is actually an AE or CE, such an effect will always amount to a violation of IIA.

${ }^{5}$ As we explain below, these models cannot be directly applied to our setup, since our set of feasible payoffs is not convex. We instead apply the appropriate extensions of these solutions, from Mariotti (1998) and Nagahisa and Tanaka (2002).
} 
contract. One source of focality can be desirable money payoffs (equal or total earnings maximizing, or some combination thereof). Based on existing research (see, e.g., Roth and Murnighan, 1982; Isoni et al., 2014; Luhan et al., 2017; Galeotti et al., 2019), we expect such payoff focality to influence the agreement. Payoff based notions such as equality and total earnings are cardinal properties. Our hypothesis is that what we call relational focality, namely salient properties of a contract based on ordinal (rank-based) comparisons (such as a contract being a compromise between others, or dominating another contract) also serve to make a contract focal, and this gives rise to the $\mathrm{CE}$ and $\mathrm{AE} .{ }^{6}$ We expect that if a contract is both payoff and relationally focal, then it is highly likely to be agreed on. It is perhaps more interesting to consider how payoff and relational focality interact when they suggest different agreements, and the purpose of the paper is to shed empirical light on this relationship. ${ }^{7}$

We conducted two within-subject experiments. In Experiment 1, we explore different games where we vary the payoffs, and hence the payoff focality, of the base contracts $A$ and $B$, and measure if, by adding the $C$ contract, making one of the base contracts a compromise or dominant has a significant effect on the observed distribution of agreements. The findings from the first experiment led us to conduct a second experiment that employed the same design and procedures as Experiment 1 but with a different list of games to test the robustness of the CE and AE. The list includes both old (from Experiment 1) and new games. The main finding of these experiments is that there are significant AEs and CEs in our bargaining environment, but only under certain conditions on contracts $A$ and $B^{\prime}$ s (and sometime $C^{\prime}$ s) payoffs. Relational focality is thus constrained by payoff focality. Our interpretation is that it is only when payoff focality is relatively weak that relational focality, and hence the $\mathrm{CE}$ and $\mathrm{AE}$, significantly manifest themselves. We now provide more detail on our findings.

Significant CE: We observe a significant $C E$ when the two base contracts $A$ and $B$ are 'equally unequal' (namely located symmetrically around the 45 degree line), and there is not too much conflict among the players about which one to agree to.

Second, if neither $A$ nor $B$ offers equal payoffs (and they are not symmetric around the 45 degree line), then a significant $C E$ arises when the target contract (the one that is made a compromise by adding $C$ ) is the least unequal of the contracts.

Third, there is a significant CE even when the target contract is Pareto efficient and offers the players exactly the same payoffs, as long as the total earnings offered by the contract are sufficiently small. Otherwise the contract is so payoff focal that there is little 'room' for raising its popularity further by making it a compromise. In our opinion, this is a particularly important finding: relational focality can matter for a contract even when that contract already possesses a

\footnotetext{
${ }^{6}$ There is also a literature on focality based on purely contextual properties ('labels'); see for example Schelling (1960); Mehta et al. (1994); Crawford et al. (2008); Bardsley et al. (2010); Isoni et al. (2013, 2014); Faillo et al. (2017); Isoni et al. (2020).

${ }^{7}$ These sources of focality can also be thought of as affecting the players' relative bargaining power, in that they influence what bargaining positions a player can credibly take up (see also Roth and Murnighan, 1982; Roth, 1985; Bolton and Karagözoğlu, 2016). According to this view, the AE and CE play a role in bargaining because they affect a player's bargaining power. We thank a referee for drawing our attention to this relationship.
} 
very strong property of offering perfectly equal and efficient payoffs.

Significant $A E$ : We observe a significant $\mathrm{AE}$ when the target contract is the less unequal base contract, and neither the $C$ contract nor the target are exactly equal. If the base contracts are symmetric around the 45 degree line, and hence equally payoff focal, the AE is less strong than the CE.

The rest of the paper is organized as follows. In Section 2 we describe the related literature. We define the AE and CE in bargaining in Section 3. Section 4 introduces the experimental bargaining games, the experimental design and logistics of the first experiment. Section 5 describes our predictions and hypotheses. The experimental findings of the first experiment are reported in Section 6. Section 7 contains the experimental games and design of the second experiment. Section 8 presents the main results of this second study. Section 9 discusses the findings, and suggests some future research. We conclude in Section 10. The Online Appendix contains the experimental instructions, additional theory, and supplementary data analysis.

\section{Related Literature}

While there have been many studies of the AE and CE in individual choice settings (for an overview, see Frederick et al., 2014; Lichters et al., 2015, 2016), ${ }^{8}$ we are aware of only few studies of these effects in interactive settings. Galeotti et al. (2019) considered the trade-off between equality and efficiency in a bargaining setting. Their games vary the severity of the trade-off between equality and total earnings maximization. Some of their games are two-sided, consisting of one equal contract and two symmetrically unequal contracts, while others are one-sided, consisting of one equal and one unequal contract. In comparing one-sided and two-sided games, they observed that the equal contract is more likely to be agreed upon in two-sided games, a finding they interpret as a CE. The AE was not investigated. ${ }^{9}$ The current paper launches a systematic investigation of the $\mathrm{CE}$ and $\mathrm{AE}$ in bargaining.

We are aware of two other investigations of the AE (but not $\mathrm{CE}$ ) in interactive situations. Colman et al. (2007) and Amaldoss et al. (2008) consider one-shot simultaneous-move games with 'strategic asymmetric dominance'. This means that a player has a strategy $x$ that is strictly or weakly dominated by just one of the other strategies, $y$. There is a strategic asymmetric dominance effect if the presence of $x$ makes the player more likely to choose $y$. Since $x$ is dominated by $y$ for an individual player, their AE is close to the one found in individual choice.

The main purpose of our experiment is to collect data rather than to develop and test a theory of the $\mathrm{AE}$ and $\mathrm{CE}$ in bargaining. It may nonetheless be useful to note that some of the proposed explanations for the $\mathrm{AE}$ and $\mathrm{CE}$ in individual choice settings ${ }^{10}$ can be relevant for bargaining.

\footnotetext{
${ }^{8}$ Most of the empirical studies use hypothetical choice methods. Some exceptions are Simonson and Tversky (1992), Doyle et al. (1999), Herne (1999), Beauchamp et al. (2019), de Haan and van Veldhuizen (2015), and Lichters et al. (2017). These studies find significant effects with incentivized choice. Some criticisms of the existing studies are raised in Frederick et al. (2014), Yang and Lynn (2014), and Lichters et al. (2015).

${ }^{9}$ Some of the games we use are the same as in Galeotti et al. (2019), and we compare the findings in Section 6 and 8.

${ }^{10}$ These explanations include reason-based choice (see Simonson, 1989; Shafir et al., 1993), where the decision maker
} 
For example, in a multi-attribute choice set-up Poterack (2015) and Tserenjigmid (2019) let the minimum value along each dimension serve as a reference point. In a bargaining context the addition of a decoy changes the minimum payoff from an agreement for one but not the other player (cf Figure 2). If players resist agreeing to their least favorable contract, this would create an effect in the direction of the $\mathrm{CE}$ and AE. ${ }^{11}$ Future work can develop and experimentally test such theories of the $\mathrm{AE}$ and $\mathrm{CE}$ in bargaining.

\section{The Attraction and Compromise Effects in Bargaining}

A contract specifies how much money Player 1 and 2 gets. Player 1 and 2 negotiate either over a set of two contracts, denoted $S=\{A, B\}$, or over a set of three contracts, $T=\{A, B, C\}$. The only difference is thus whether contract $C$ is feasible or not. We refer to the contracts in $S$ as the base contracts. The bargaining game based on a given contract set is referred to simply as the game $(\mathrm{G})$. We refer to the game based on contract set $S$ as the base game (BG). Contract $C$ is referred to as the decoy, and the base contract that the decoy is intended to make more focal is the target.

Denote by $p_{i}^{S}$ the proportion of bargaining pairs who agree to contract $i$, where $i=A, B$, when the contract set is $S=\{A, B\}$. Similarly, let $p_{j}^{T}$ denote the proportion of bargaining pairs who agree to contract $j=A, B, C$ when the contract set is $T=\{A, B, C\}$. All these proportions are calculated out of all interactions, including those that ended in disagreement. We define the AE and $\mathrm{CE}$ in terms of these aggregate contract agreement proportions.

In what follows we assume that contract $B$ is the target (as in Figure 2). We say that a contract strictly dominates another if the former offers each player a strictly higher amount of money than the latter, and that it is a compromise if for each player its money payouts are the second highest, while each of the other contracts gives one player its highest payoff and the other its lowest.

Definition 1. (Attraction Effect, AE) Suppose the decoy $C$ is strictly dominated by $B$, but not by $A$. The $A E$ arises when $p_{B}^{T}>p_{B}^{S}$.

Definition 2. (Compromise Effect, CE) Suppose the decoy $C$ makes $B$ a compromise. The Compromise Effect arises when $p_{B}^{T}>p_{B}^{S}$.

We detect $\mathrm{AE}$ and $\mathrm{CE}$ in the data by comparing the proportions of agreements on the target contract when the contract set is $S$ and when it is $T$. If the latter proportion is significantly larger than the former, we reject the null hypothesis in favor of the alternative hypothesis, that there is an AE or CE.

when faced with a difficult choice looks for reasons that allow him or her to make a decision; such reasons can be based on dominance or compromise. Other explanations rely on reference-dependent individual choice, where the attractiveness (utility) of a choice alternative is assumed to depend not only on its own absolute properties, but also on how it is related to other alternatives (see for example Wedell, 1991; Simonson and Tversky, 1992; Tversky and Simonson, 1993; Kivetz et al., 2004; Bordalo et al., 2013; Cunningham, 2013; Kőszegi and Szeidl, 2013; Masatlioglu and Uler, 2013; Ok et al., 2015; Poterack, 2015; Tserenjigmid, 2019; Bushong et al., 2020; Castillo, 2020).

${ }^{11}$ We thank a reviewer for drawing our attention to this possibility. 


\section{Experiment 1}

\subsection{The Bargaining Games}

We collected data from 22 games, shown in Table 1 and Figure 3. There are four Base Games (BG), 1, 4,7 , and 16 (marked in grey in the table), each with contract sets $S=\{A, B\}$. In Game 1 (BG1) the two contracts are 'symmetrically unequal'. In BG4 there is an equal and total earnings maximizing contract $(B)$. BG7 is similar to BG4, except that the equal contract is Pareto efficient but not total earnings maximizing. Finally, BG16 has a contract that offers nearly equal payoffs. By adding different $C$ contracts to the base games, we can assess how the strength of the AE and CE depends on the nature of the added contract $C$, and how these effects vary across different base games.

We also collected data for two games, G15 and G22, where the $C$ contract was strictly dominated by both base contracts. We thought it would be interesting to see if the presence of such clearly inferior contracts could still exert a significant influence on the bargaining outcome.

\begin{tabular}{|c|c|c|c|c|c|}
\hline \multirow{2}{*}{ Game } & \multicolumn{3}{|c|}{ Contracts } & \multirow{2}{*}{ Target } & \multirow{2}{*}{ Hypothesized effect } \\
\hline & $\mathbf{A}$ & B & C & & \\
\hline 1 & $(40,60)$ & $(60,40)$ & & - & - \\
\hline 2 & $(40,60)$ & $(60,40)$ & $(80,20)$ & B & Compromise \\
\hline 3 & $(40,60)$ & $(60,40)$ & $(50,30)$ & B & Attraction \\
\hline 4 & $(40,120)$ & $(80,80)$ & & - & - \\
\hline 5 & $(40,120)$ & $(80,80)$ & $(20,140)$ & A & Compromise \\
\hline 6 & $(40,120)$ & $(80,80)$ & $(20,100)$ & $\mathrm{A}$ & Attraction \\
\hline 7 & $(40,120)$ & $(60,60)$ & & - & - \\
\hline 8 & $(40,120)$ & $(60,60)$ & $(5,155)$ & A & Compromise \\
\hline 9 & $(40,120)$ & $(60,60)$ & $(30,130)$ & $\mathrm{A}$ & Compromise \\
\hline 10 & $(40,120)$ & $(60,60)$ & $(120,40)$ & B & Compromise \\
\hline 11 & $(40,120)$ & $(60,60)$ & $(155,5)$ & B & Compromise \\
\hline 12 & $(40,120)$ & $(60,60)$ & $(70,40)$ & B & Compromise \\
\hline 13 & $(40,120)$ & $(60,60)$ & $(30,110)$ & $\mathrm{A}$ & Attraction \\
\hline 14 & $(40,120)$ & $(60,60)$ & $(50,50)$ & B & Attraction \\
\hline 15 & $(40,120)$ & $(60,60)$ & $(30,30)$ & B & Decoy strictly dominated by A and B \\
\hline 16 & $(40,120)$ & $(65,55)$ & & - & - \\
\hline 17 & $(40,120)$ & $(65,55)$ & $(30,130)$ & $\mathrm{A}$ & Compromise \\
\hline 18 & $(40,120)$ & $(65,55)$ & $(120,40)$ & B & Compromise \\
\hline 19 & $(40,120)$ & $(65,55)$ & $(50,50)$ & B & Attraction \\
\hline 20 & $(40,120)$ & $(65,55)$ & $(55,45)$ & $\mathrm{B}$ & Attraction \\
\hline 21 & $(40,120)$ & $(65,55)$ & $(30,110)$ & $\mathrm{A}$ & Attraction \\
\hline 22 & $(40,120)$ & $(65,55)$ & $(30,30)$ & $\mathrm{B}$ & Decoy strictly dominated by A and B \\
\hline
\end{tabular}

Note: Base games $(1,4,7,16)$ are in grey.

Table 1: The bargaining games for Experiment 1. 


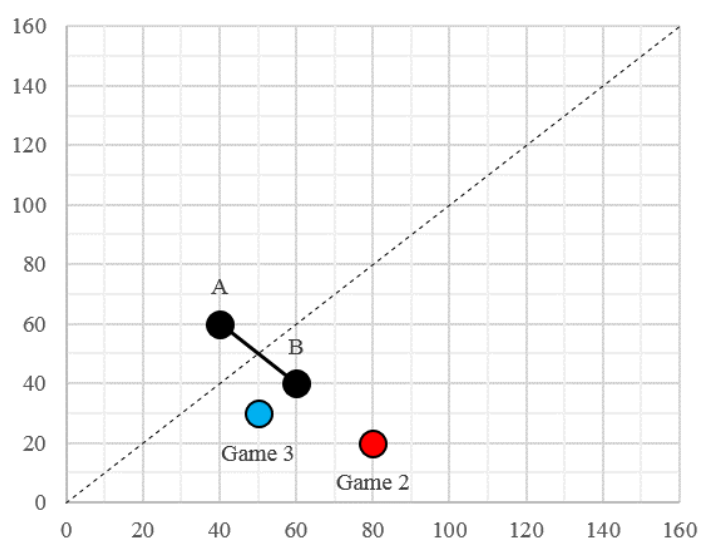

(a) Games 1-3.

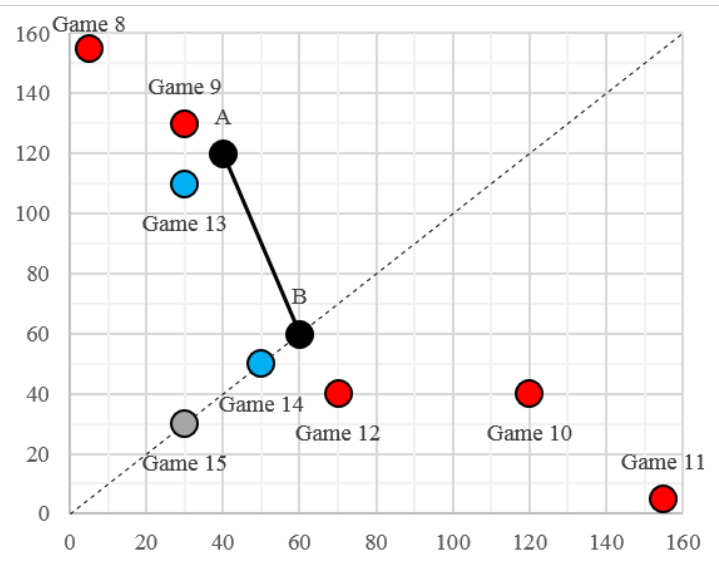

(c) Games 7-15.

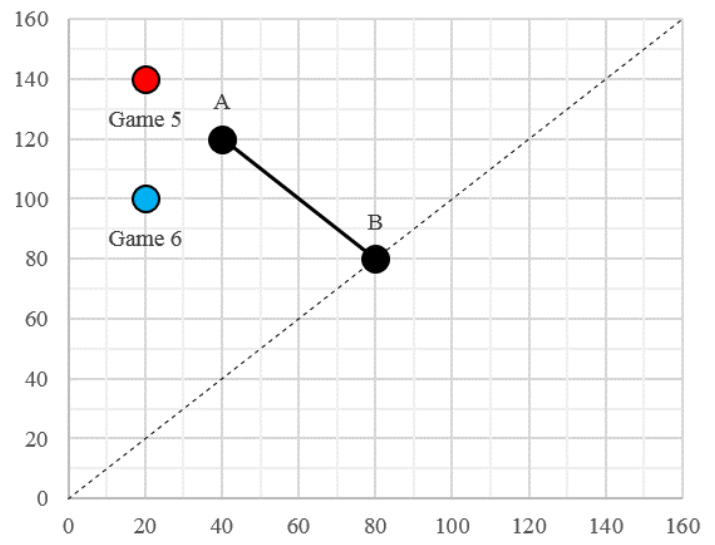

(b) Games 4-6.

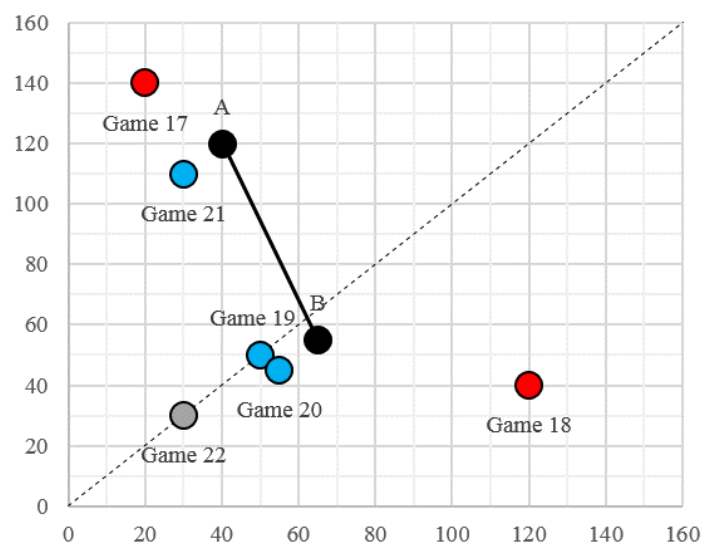

(d) Games 16-22.

Figure 3: Graphical illustration of the bargaining games.

Note: The axes measure (in experimental points) how much each Person ( 1 and 2$)$ gets for a given agreed contract. The base contracts $A$ and $B$ are in black. The decoy contract $C$ is in red (blue) when it is hypothesized to generate a $C E$ (AE). It is in grey when it is dominated by both $A$ and $B$.

\subsection{Experimental Design and Procedures}

The experiment involved 17 sessions with 16 participants per session ( 272 participants in total). We used the software $z$-Tree (Fischbacher, 2007) to program and conduct the experiment, and ORSEE (Greiner, 2015) to recruit the participants. Subjects earned on average $£ 13$ (including a show-up fee of $£ 4$ ) and each session lasted just below an hour.

Upon arriving to the lab, subjects were allocated to different desks separated by partitions. They received printed instructions (see Online Appendix) which were read aloud by the experimenter. Each subject encountered the 22 bargaining games in a different order. The design is thus within-subject, providing a more powerful testbed for the hypothesized effects. Subjects did not know the content of the 22 games in advance, and only knew that they would be re-matched after 
each game. ${ }^{12}$ Since different subjects encounter the games in a different order and are re-matched in every round, we do not expect strong learning or order effects in the data that would bias our comparisons across games, given similar experimental setups (see e.g. Andreoni and Miller, 2002; Isoni et al., 2014). Additional data analysis reported in the Online Appendix supports this conclusion.

In each game that a subject encountered, one randomly selected subject was referred to as Person 1 and the other as Person 2. Hence, a subject could be Person 1 in some games, and Person 2 in others. We used these player labels to simplify the description of and reference to the contracts. When two subjects were matched, it was randomly decided which feasible payoffs were assigned to Person 1 or 2. As an example, G4 came in two versions: Person 1 has feasible payoffs 40 and 80 (so 2 has 80 and 120), and Person 1 has payoffs 80 and 120 (2 has 40 and 80). We analysed the data and found no significant effect of the labels on behavior. In particular, the earnings of subjects labeled Person 1 and 2 are not significantly different (Wilcoxon signed-rank test, $p=0.687$ ). ${ }^{13}$

Each pair of subjects were presented with a set of either two or three contracts. The contracts were displayed, in the same random order, on the matched subjects' computer screens. In each game subjects were given 120 seconds to negotiate. They made contract proposals by clicking with their mouse on a contract, and could write free-form chat messages to each other. Subjects could write as many or as few messages as they wanted. They were asked not to reveal their identity, physically threaten the other subject, or discuss what might happen outside the lab. Subjects were informed that failure to comply would result in exclusion.

Figure 4 shows the computer screen the subjects saw. Note that the contracts were not given any particular labels. In order to reach an agreement, subjects had to click on the same contract. An agreement was binding and could not be changed. As long as subjects had not clicked on the same contract, they could withdraw their contract proposal or replace it with a new one, in real time and as many times as they wanted. Subjects were also free to make no proposals at all. If no agreement was reached before the end of the 120 seconds, the two paired subjects earned no points from that game.

\footnotetext{
${ }^{12}$ We designed the matching protocol algorithm in order to minimize the re-matching between the same participants in order to curtail repeated-game effects.

${ }^{13}$ Similarly, we find no labeling effect on who first starts the chat $(p=0.421)$ or sends a proposal $(p=0.461)$. When we analyze the data, we thus pool the data across player labels.
} 


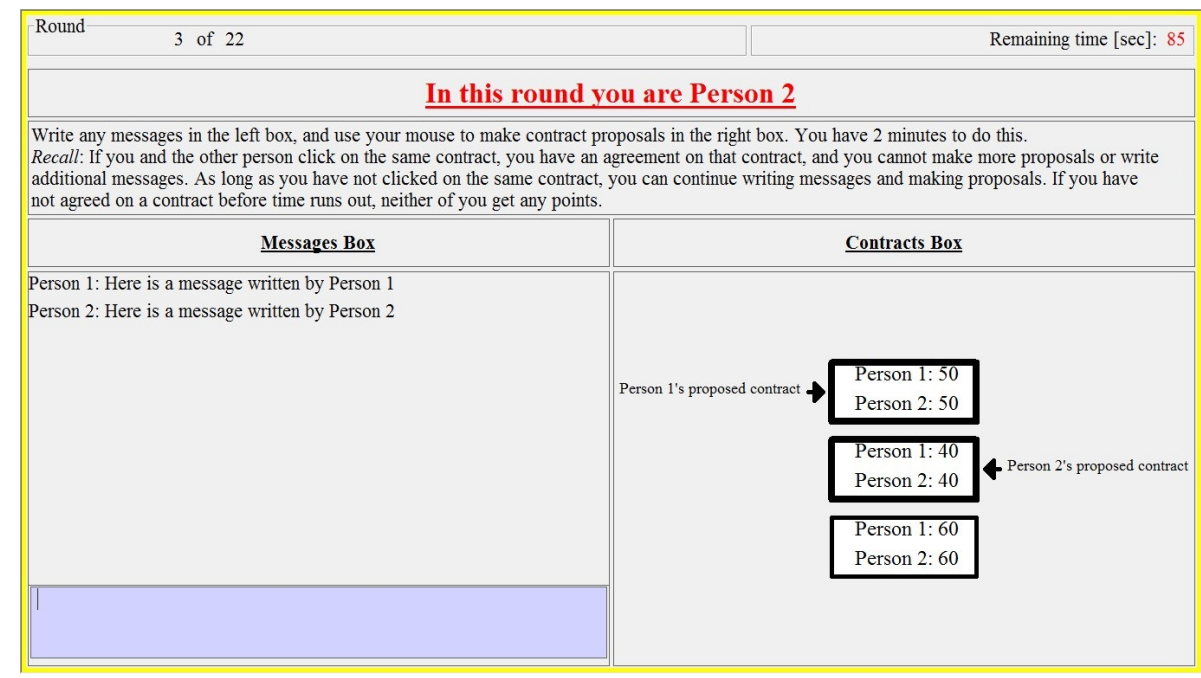

Figure 4: Decision screen.

At the end of the experiment, the computer randomly selected three of the twenty-two rounds (the same for all subjects in a given session) for payment. Points were converted to pounds at the exchange rate of 20 points $=£ 1$. Paying for the outcome of a selected number of periods rather than just one is an alternative method to the "pay-one" approach but with the advantage of equilibrating more the payment across participants and limiting the impact of very unlucky draws in the final earnings (see Charness et al., 2016).

\section{Theory and Hypotheses}

Our unstructured bargaining protocol gives the subjects complete freedom to make offers whenever they wish. This, with the fact that communication via chat messages is allowed, and that an agreement can be made binding, makes predictions from cooperative bargaining theory (see Thomson, 1994) relevant.

\subsection{The IIA Axiom}

We start by considering what the well-known axiom of Independence of Irrelevant Alternatives (IIA) interpreted as a positive statement about what will (not) happen in an actual bargaining situationimplies for the AE and CE. Nash (1950) defined IIA for single-valued solution concepts; we use the analogous definition for set-valued solution concepts ${ }^{14}$ which can be found in Mariotti (1998). Let $\varphi(T)$ be the set of contracts predicted by a solution when the set of contracts is $T$.

Definition 3. (IIA) If $S \subset T$ and $\varphi(T) \cap S \neq \varnothing$, then $\varphi(S)=\varphi(T) \cap S$.

\footnotetext{
${ }^{14}$ In a bargaining situation with a finite set of contracts, theoretical predictions are potentially set valued. For example in a situation like BG1, where the set of contracts is $\{(40,60),(60,40)\}$, it is not possible for a solution to be symmetric, Pareto efficient (which rules out the disagreement point $(0,0)$ ) and single valued. Any symmetric and Pareto efficient solution consists of $\{(40,60),(60,40)\}$.
} 
There is a quite simple intuition behind IIA. If the bargainers find a contract, say $B$, attractive enough to possibly agree on it when there is a large set of alternative contracts (contract set $T$ ), then the bargainers should still find $B$ sufficiently attractive when some of the 'rival' contracts are not available (contract set $S$ ). Furthermore, contracts that were ruled out in $T$ should still be ruled out in $S$, since (some of the) contracts that were considered more attractive are still available.

We interpret set-valued predictions as a prediction that the final agreement will lie in the set. Without adding further restrictions on the probability of agreements on different contracts, an observation that there are significantly more agreements on B in G2 and G3 than in BG1 is not inconsistent with IIA. In order to deal with this indeterminacy, we appeal to the principle of insufficient reason and introduce the following auxiliary assumption.

Assumption 1. If the solution contains two or more contracts, then each of them is equally likely to be agreed upon.

In terms of our data, IIA together with assumption 1 predicts that

$$
p_{A}^{S} \geq p_{A}^{T} \text { and } p_{B}^{S} \geq p_{B}^{T}
$$

which clearly rules out both the AE and CE. ${ }^{15}$

Hypothesis 1. (IIA) There are neither AE nor CE in any of the bargaining games.

Note that the IIA axiom is silent on what contracts will actually be agreed upon: all it predicts is that the frequency of agreements on any given contract cannot decrease as the set of available contracts shrinks. We also discuss the predictions of the leading solution concept in cooperative bargaining theory, namely the Nash Bargaining Solution (Nash, 1950, in what follows referred to as NBS), which satisfies the IIA axiom. The original NBS assumed that the set of feasible payoffs is convex, which does not hold in our finite set-up (recall that players cannot make a binding agreement to a lottery between contracts). We therefore consider the extension of the original solution to a finite set of feasible payoffs by Mariotti (1998). ${ }^{16}$ His solution selects the contract(s) with the highest Nash product. Suppose bargainers are rational, self-regarding, and risk neutral, and that this is common knowledge. ${ }^{17}$ Then some straightforward calculations reveal that the NBS predicts contracts $\{A, B\}$ in BG1-G3, contract $B$ in BG4-G6, and contract $A$ in BG7-G22, except G10 and G18, where the prediction is $\{A, C\}$.

\footnotetext{
${ }^{15}$ Since in our experiment subjects are rematched from round to round, we do not observe the same pairs bargaining over $S=\{A, B\}$ and $T=\{A, B, C\}$, so we expect IIA to hold only in a probabilistic sense. Suppose IIA holds for each possible pair in the population; then any pair that agrees on a base contract in contract set $T$ agrees on the same contract in set $S$ (and any pair that is equally likely to agree to two or more contracts in contract set $T$ does the same in $S$ ). The actual frequency of agreements on the base contract can occasionally be lower in $S$ than in $T$ because we are looking at a sample rather than at all possible pairs in the population, but it should not be systematically lower. If it is, IIA is violated.

${ }^{16}$ Another extension of the NBS to a non-convex domain is Conley and Wilkie (1996), but they assume that the set is comprehensive, which means that the players can dispose of utilities (see Thomson, 1994). This is not possible in our experiment.

${ }^{17}$ The restrictiveness of these assumptions is discussed below.
} 
Mariotti's solution never generates an AE, since a dominated contract always has a smaller Nash product than the contract that dominates it. The same is true for the CE if there is only one contract that maximizes the Nash product. With the addition of Assumption 1, Mariotti's model predicts that there will never be a CE. ${ }^{18}$

While contracts predicted by the NBS clearly depend on our assumption that agents are selfinterested and risk neutral, the prediction that there is no AE and no CE holds for other preferences, e.g. inequity averse or social welfare preferences (see Fehr and Schmidt, 1999; Charness and Rabin, 2002), provided that the utility a player gets from a contract depends only on that contract and not on what other contracts are available.

\subsection{Bargaining Solutions that Predict a Compromise and/or Attraction Effect}

According to the Fallback Bargaining Solution (see Brams and Kilgour 2001; Kıbris and Sertel 2007; de Clippel and Eliaz 2012; in what follows referred to as FBS) each player has a strict ranking of the contracts. The players first consider if a contract is ranked first by both players; if so, they agree to it. Otherwise, they look for a contract that is ranked either first or second by both players. If such a contract exists, it becomes the agreement. If not, they consider if there are contracts that are ranked first, second, or third, by each player, and so on.

As de Clippel and Eliaz (2012) point out, the FBS generates an AE and CE. Suppose Player 1's ranking is $A \succ B$, and 2 has the opposite ranking. If they bargain over $S$, the fallback solution is $\{A, B\}$, and by Assumption 1 each alternative is equally likely to be agreed upon. Suppose then the set of contracts is $T$ and that contract $C$ is dominated by $B$ (but not by $A$ ). Player 1's ranking is $B \succ C \succ A$, while 2's is $A \succ B \succ C$. The decoy drives a wedge between Player 1's ranking of $A$ and $B$, and $A$ is now relatively worse for Player 1 than before. The FBS is $B$. Similarly, there can be a CE. If a contract $C$ is added such that Player 1's ranking is $C \succ B \succ A$, and 2's is $A \succ B \succ C$, the solution is again $B$. For Player 1 the decoy pushes $A$ to the bottom.

Hypothesis 2. (Fallback Bargaining Solution): There is an AE and a CE in all games.

An alternative to the NBS is the Kalai and Smorodinsky (1975) bargaining solution (henceforth KSBS), extended to a finite set of feasible payoffs by Nagahisa and Tanaka (2002). Their solution selects the contract(s) that maximize the payoff of the player getting the lower proportion of his or her maximal possible ('ideal') payoff. Since adding a strictly dominated decoy does not affect the ideal point, this solution never predicts an $\mathrm{AE}$, but would predict a $\mathrm{CE}$ in some of our games (see Online Appendix for more details). The Ordinal Egalitarian Solution (OES), due to Conley and Wilkie (2012), generates a CE in all possible cases. It never predicts an AE.

\footnotetext{
${ }^{18}$ The Nash product of a contract does not depend on what other contracts are available. Thus, either i) $C$ does not maximize the Nash product in $T$, in which case the solutions for $S$ and $T$ must be identical (and, if the solution is set-valued, frequencies of individual contracts are unchanged because of assumption 1), ii) $C$ is the only contract that maximizes the Nash product in $T$, or iii) $C$ and one or more other contracts maximize the Nash product in $T$, in which case any contract in the solution for $S$ must still be part of the solution for $T$, and its predicted frequency must decline by Assumption 1.
} 


\subsection{Relational Versus Payoff Focality}

The FBS (and indeed the CE and AE themselves) are defined on the basis of purely ordinal information, i.e., only the players' ranking of the contracts matters. This is what we above refer to as relational focality. However, cardinal properties, such as equality of payoffs and total earning maximization, are also important sources of focality (payoff focality).

Consider first the case where there is a base contract, say $B$, with equal and total-earnings maximizing payoffs (BG4-G6). This is the most challenging environment for the $\mathrm{CE}$ or $\mathrm{AE}$ to occur, since $B$ is likely to be strongly payoff focal. A more favorable environment is BG7-G15, where there is an equal contract competing with an unequal but total-earnings maximizing contract, and targeting one of the two contracts with a decoy may tilt the balance in its favor.

In games BG16-G21, the equal payoff base contract is replaced with one offering nearly equal payoffs; this weakens its payoff focality and hence leaves more room for relational focality and the CE or AE. Finally, in BG1-G3 the two base contracts are symmetrically unequal, and since the contracts are therefore equally payoff focal we expect this environment to be the most favorable to the $\mathrm{CE}$ and $\mathrm{AE}$.

We also wished to investigate if the strength of the CE depends on how attractive the decoy itself is. We conjectured that an equal payoff decoy may be more effective than an unequal decoy, since bargainers may notice an equal contract first and then look for Pareto improvements on the decoy. This question is addressed by comparing BG16-G19 and BG16-G20. The target is the same and offers unequal payoffs, while the decoy is either equal or nearly equal. Similarly, we conjectured that a very extreme decoy-where, by extreme, we mean how unequal the decoy iswould be more likely to be ignored (because of its implausibility as an agreement). We examine this by comparing G8 and G9 (where the compromise is contract $A$ ), and G10, G11, and G12 (the compromise is $B$ ).

Finally, we wished to consider games with decoys $C$ that were dominated not just by a single but both base contracts. The conditions for the $\mathrm{AE}$ are not satisfied in this case (the $\mathrm{AE}$ requires $\mathrm{C}$ to be dominated by only one base contract). A natural null hypothesis is that there is no effect of adding these contracts. An alternative hypothesis is that such $C$ contracts can still affect behavior by being closer to one of the base contracts, and in this sense still act as a 'decoy' for that base contract. We test this by comparing BG7 with G15, and BG16 with G22.

\section{Findings of Experiment 1}

\subsection{Overview}

Table 2 shows descriptive statistics for the 22 games. The feasible contracts for each game are shown in the 'Contracts' column, followed by the percentage of bargaining pairs who did not reach an agreement, and who agreed on the contract A, B, and C, respectively (columns 'Disagree', 'Agree on A', 'Agree on B', and 'Agree on C'). Recall that since we found no effects of labels (Person 
1 vs 2) on behavior, we pool the data across player labels 1 and $2 .^{19}$

Table 2 also shows how long it on average took for people to reach an agreement ('Time to agree' column). Note that pairs who disagreed are excluded from this average.

\begin{tabular}{|c|c|c|c|c|c|c|c|c|}
\hline \multirow{2}{*}{ Game } & \multicolumn{3}{|c|}{ Contracts } & \multirow{2}{*}{ Disagree } & \multirow{2}{*}{$\begin{array}{c}\text { Agree } \\
\text { on } A\end{array}$} & \multirow{2}{*}{$\begin{array}{c}\text { Agree } \\
\text { on B }\end{array}$} & \multirow{2}{*}{$\begin{array}{l}\text { Agree } \\
\text { on C }\end{array}$} & \multirow{2}{*}{$\begin{array}{c}\text { Time to } \\
\text { agree (in sec.) }\end{array}$} \\
\hline & A & B & C & & & & & \\
\hline 1 & $(40,60)$ & $(60,40)$ & & $8.08 \%$ & $45.96 \%$ & $45.96 \%$ & $0 \%$ & 89.93 \\
\hline 2 & $(40,60)$ & $(60,40)$ & $(80,20)$ & $6.62 \%$ & $35.29 \%$ & $55.15 \%$ & $2.94 \%$ & 90.21 \\
\hline 3 & $(40,60)$ & $(60,40)$ & $(50,30)$ & $7.35 \%$ & $38.23 \%$ & $52.21 \%$ & $2.21 \%$ & 88.38 \\
\hline 4 & $(40,120)$ & $(80,80)$ & & $0 \%$ & $2.21 \%$ & $97.79 \%$ & $0 \%$ & 34.71 \\
\hline 5 & $(40,120)$ & $(80,80)$ & $(20,140)$ & $0.74 \%$ & $1.47 \%$ & $97.05 \%$ & $0.74 \%$ & 35.63 \\
\hline 6 & $(40,120)$ & $(80,80)$ & $(20,100)$ & $0 \%$ & $1.47 \%$ & $98.53 \%$ & $0 \%$ & 37.09 \\
\hline 7 & $(40,120)$ & $(60,60)$ & & $0.74 \%$ & $7.35 \%$ & $91.91 \%$ & $0 \%$ & 63.67 \\
\hline 8 & $(40,120)$ & $(60,60)$ & $(5,155)$ & $5.15 \%$ & $8.09 \%$ & $86.76 \%$ & $0 \%$ & 63.28 \\
\hline 9 & $(40,120)$ & $(60,60)$ & $(30,130)$ & $1.47 \%$ & $11.03 \%$ & $87.5 \%$ & $0 \%$ & 53.38 \\
\hline 10 & $(40,120)$ & $(60,60)$ & $(120,40)$ & $1.47 \%$ & $3.68 \%$ & $91.17 \%$ & $3.68 \%$ & 49.04 \\
\hline 11 & $(40,120)$ & $(60,60)$ & $(155,5)$ & $0.74 \%$ & $7.35 \%$ & $91.17 \%$ & $0.74 \%$ & 52.86 \\
\hline 12 & $(40,120)$ & $(60,60)$ & $(70,40)$ & $2.21 \%$ & $4.41 \%$ & $91.17 \%$ & $2.21 \%$ & 51.23 \\
\hline 13 & $(40,120)$ & $(60,60)$ & $(30,110)$ & $3.68 \%$ & $9.56 \%$ & $86.76 \%$ & $0 \%$ & 54.19 \\
\hline 14 & $(40,120)$ & $(60,60)$ & $(50,50)$ & $3.68 \%$ & $5.88 \%$ & $90.44 \%$ & $0 \%$ & 44.34 \\
\hline 15 & $(40,120)$ & $(60,60)$ & $(30,30)$ & $2.94 \%$ & $7.35 \%$ & $89.71 \%$ & $0 \%$ & 52.19 \\
\hline 16 & $(40,120)$ & $(65,55)$ & & $7.35 \%$ & $17.65 \%$ & $75 \%$ & $0 \%$ & 73.51 \\
\hline 17 & $(40,120)$ & $(65,55)$ & $(30,130)$ & $6.62 \%$ & $17.65 \%$ & $75 \%$ & $0.73 \%$ & 65.04 \\
\hline 18 & $(40,120)$ & $(65,55)$ & $(120,40)$ & $5.88 \%$ & $5.15 \%$ & $87.5 \%$ & $1.47 \%$ & 50.35 \\
\hline 19 & $(40,120)$ & $(65,55)$ & $(50,50)$ & $4.41 \%$ & $8.82 \%$ & $72.80 \%$ & $13.97 \%$ & 57.58 \\
\hline 20 & $\begin{array}{l}(40,120) \\
\end{array}$ & $(65,55)$ & $(55,45)$ & $0.74 \%$ & $11.03 \%$ & $88.23 \%$ & $0 \%$ & 55.92 \\
\hline 21 & $(40,120)$ & $(65,55)$ & $(30,110)$ & $2.21 \%$ & $17.65 \%$ & $79.41 \%$ & $0.73 \%$ & 74.83 \\
\hline 22 & $(40,120)$ & $(65,55)$ & $(30,30)$ & $2.21 \%$ & $12.5 \%$ & $84.56 \%$ & $0.73 \%$ & 61.35 \\
\hline
\end{tabular}

Notes: For each game there are 136 observations (number of pairs). Base games are shaded in grey. The contract labels $A, B$, and $C$ were not used in the experiment.

Table 2: Aggregate bargaining outcomes (Experiment 1).

A visual representation of how agreements (and disagreements) vary between each BG1, BG4, BG7, and BG16 and the other games is given in Figure 5.

To test our hypotheses, we conduct Wilcoxon signed-rank tests using session averages as the units of observation. ${ }^{20}$ Our hypotheses predict an effect in a particular direction (the AE and CE are directional effects), except in cases where $\mathrm{C}$ is dominated by both $\mathrm{A}$ and $\mathrm{B}$. Hence, all statistical tests regarding AEs and CEs are one-tailed (this is specified when we report the results). In all the other cases where we do not have a-priori or directional hypotheses, the tests are two-tailed. Significance is evaluated at the $5 \%$ level, unless otherwise specified.

We think of the $\mathrm{AE}$ and $\mathrm{CE}$ as effects that benefit the target, so in order to claim that an $\mathrm{AE}$ or $\mathrm{CE}$ has occurred, we require that the relative frequency of agreements on the target increases, computed as a fraction of all interactions, not just those interactions that end in an agreement.

\footnotetext{
${ }^{19}$ Note that G1 and G10 are symmetric, so as a result of the pooling the agreement proportions on the unequal payoff contracts are identical.

${ }^{20}$ In each session, subjects are exposed to the same 22 games. Hence, we have 22 multiple related observations for each session. This is why we use Wilcoxon signed-rank tests. As a robustness check, we also conducted parametric tests. In particular, we checked the statistical significance of marginal effects computed from logit regressions on the pair-level data. The dependent variable is a dichotomous variable equal to one if the pair agrees on the targeted contract, while the independent variables are dummies for the different games. Standard errors are clustered at the session level. The results of parametric and non-parametric tests are qualitatively similar. Full details are available from the authors upon request.
} 

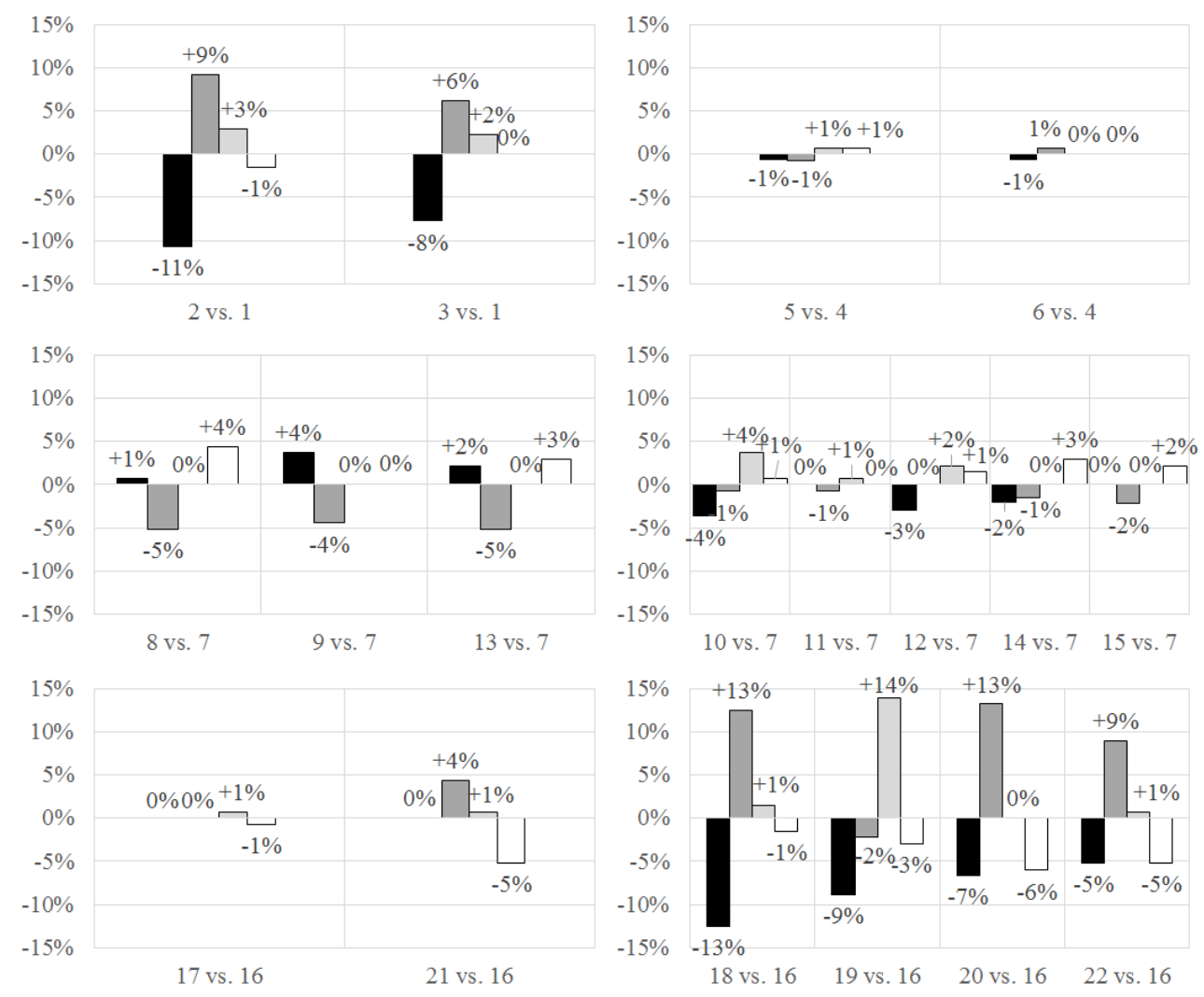

- A $\square \mathrm{B} \square \mathrm{C} \square$ Disagree

Note: the difference in the agreement rate on $C$ between a game and its corresponding base game is obtained by setting the agreement rate on $C$ equal to zero in the base game.

Figure 5: Changes in agreements on $A, B, C$ and disagreements for each game, compared to the base game

Intuitively, in order for $B$ to be favored by the addition of $C, B$ should become more popular overall, not just as a fraction of the interactions that ended in an agreement. For example, if $B$ is a job applicant we think of the introduction of $C$ as favouring $B$ only if $B$ becomes more likely to be hired overall (not just conditional on the vacancy being filled) as a result of $C$ being added to the shortlist. $^{21}$

To test whether there are $\mathrm{CE}$ and $\mathrm{AE}$ in all our games pooled together (excluding those where contract $C$ is dominated by both base contracts), we calculate, for each session, the average difference in agreement on the target contract between each game and the corresponding base game. This difference is significantly greater than zero when we pool together all games where we hypothesize a CE (one-tailed test, $p=0.014$ ), and weakly significantly larger than zero when we pool together all games where we hypothesize an AE (one-tailed test, $p=0.067$ ). This suggests that both $\mathrm{CE}$ and $\mathrm{AE}$ are statistically relevant in our setting.

\footnotetext{
${ }^{21}$ We also analyzed the data including only the interactions that led to an agreement. The results are qualitatively similar. Full details are available from the authors upon request.
} 
These effects are however mainly driven by specific games (20 vs. 16 for the AE; 2 vs. 1 and 18 vs. 16 for the $C E$ ). In particular, regarding the $C E$ we find that contract $B$ is agreed on more frequently in G2 compared to BG1 (one-tailed test, $p=0.050$ ). A similar pattern is observed when we compare G18 and BG16 (one-tailed test, $p=0.007$ ). The AE in G20 is also significant (one-tailed test, $p=0.009$ ).

Finding 1. We find significant $A E$ and $C E$ in specific games, and these effects survive when we pool the data from all games together.

These findings reject IIA (Hypothesis 1), the NBS predictions, and the KSBS predictions regarding the AE. If we consider the ordinal bargaining models, the FBS and OES predictions of a CE in all games (Hypothesis 2) are clearly rejected. Moreover, neither manages to capture the observed AE pattern well (recall that the FBS predicts there should be an AE in all games, and the OES predicts the exact opposite).

\subsection{Games with an Equal Payoff Contract}

The following is immediate from Table 2.

Finding 2. There are no significant $A E$ or $C E$ in any games with an equal and total payoff maximizing contract (Games 4-6). If the equal payoff contract is not total payoff maximizing (Games 7-15), a decoy targeting the other contract has some ability to reduce agreements on the equal contract, but not to significantly increase the frequency of agreements on the target.

If we consider jointly all games with an equal contract (Games 4-6 and Games 7-15), we cannot reject the null hypothesis that the average difference in agreement on the target contract between each game and the base game is zero (one-tailed tests, $p=0.265$ for $\mathrm{CE}$ and 0.480 for AE). The same applies if we look at the games separately.

An equal and total-earnings maximizing contract is strongly payoff focal, and introducing a decoy that targets the unequal contract has no effect. The agreement rates on $A$ and $B$ are not statistically different between BG4, G5 and G6 (one-tailed tests, $p>0.1$ for all comparisons).

In BG7, subjects still agree mostly on the equal contract $B$, but less often than in BG4 ( $p=$ 0.033). This confirms the earlier finding that the payoff focality of an equal and Pareto efficient payoff contract depends on whether the contract possesses the additional property of maximizing total earnings (see Galeotti et al., 2019).

A decoy targeting the unequal contract $A$ has no effect on the target (and thus there is no CE or $\mathrm{AE}$ according to our definition), but may decrease the frequency of the equal contract. The decrease in the frequency of the equal contract is (weakly) significant in G8 and G13 but not in G9 (one-tailed tests, $p=0.051,0.079$ and 0.140 respectively). Note that the decoy itself is never chosen in these three games, and thus the decrease in the agreements on the equal contract translates into an increase in the frequency of disagreement. Finally, a decoy targeting the equal contract $B$ has 
no significant effect on either the target or the alternative contract. ${ }^{22}$

Given that the equal contract was so popular, it is not surprising that the decoys were unable to increase its frequency further. ${ }^{23}$ What may be less obvious is that the decoys did not manage to significantly increase the frequency of agreements on the other contract $(A)$ even though there is plenty of room for that to occur.

We also observe that both the NBS and KSBS systematically fail to capture the focality of the equal payoff contracts; recall that in BG7-G15 both solutions almost always predict the unequal contract $A$, while a vast majority of bargainers agree to the equal contract $B .^{24}$

\subsection{Games without an Equal Payoff Contract}

\subsubsection{Two Symmetrically Unequal Payoff Contracts}

Consider first BG1, with two unequal base contracts, $(40,60)$ and $(60,40)$. The CE is significant (cf. $\mathrm{G} 2$ ): subjects agree more on $B$ than in BG1 (one-tailed test, $p=0.050$ for $B$ ). In G3 the decoy slightly increases agreements on the target, although not significantly so at conventional levels (one-tailed test, $p=0.112$ ). The decoy also reduces the frequency of agreements on $A(p=0.070)$. Hence, there is some suggestive, but not conclusive, evidence of an AE.

Finding 3. In BG1, with two symmetrically unequal contracts, there is a significant $C E$ (G2), and some weaker evidence of an $A E$ (G3).

\subsubsection{The Case of a Nearly Equal Payoff Contract}

Let us now consider if it matters whether a contract offers exactly, or only nearly equal payoffs. We compare BG7 and BG16.

Finding 4. Observed agreements in BG16, which has a nearly equal payoff contract, are significantly different from those in BG7, which has an equal payoff contract.

In BG7 more than $90 \%$ of bargaining pairs agree to the equal contract, while only $75 \%$ do so in BG16. The agreement rate on $B$ drops by about $17 \%$ in BG16 compared to BG7 ( $p=0.001$ ), while the agreement rate on $A$ increases by about $10 \%(p=0.009)$. A similar finding for an experimental mini-Ultimatum game is reported in Güth et al. (2001). One interpretation is that the property of

\footnotetext{
${ }^{22}$ The only exception is G10, where we observe a significant drop in agreements on $A$ (one-tailed test, $p=0.020$ ). This is due to the fact that, in comparison to BG7, some agreements on $A$ are replaced by agreements on $C$.

23 The data in Galeotti et al. (2019) showed a significant CE in some (but not all) games with an equal but not totalearnings maximizing contract. Such contracts were less focal than in the current paper, and this leaves more room for their frequency to increase via a CE. This lower focality could be due to game differences (that paper included games where the equal contract offers much lower total earnings than the unequal one) or to different subject pools being used. We explore games where the equal contract offers lower payoffs in Experiment 2 (see Finding 8).

${ }^{24}$ An obvious way to improve the prediction of the models is to replace self-interested preferences with inequityaverse (Fehr and Schmidt, 1999; Bolton and Ockenfels, 2000) preferences, or allow for a mix of these different preference types. Note however that allowing for inequity aversion cannot explain any observed CE or AE according to the NBS (or any observed AE according to the KSBS).
} 
offering equal payoffs to the players confers focality over and beyond what it gets from being inequality minimizing.

Not only are agreements in BG7 and BG16 different, but the corresponding AEs and CEs are also different in magnitude. If we pool together all games with a nearly equal contract where we expect a $\mathrm{CE}$, we find that the average difference in agreement on the target contract between each game and the base game is positive and significant (one-tailed test, $p=0.001$ ). For the games where we expect an $\mathrm{AE}$, the difference is also positive but not significant $(p=0.146)$.

If we look at the games separately, the nearly equal payoffs contract $(65,55)$ can be made significantly more agreed on by making it a compromise (G18 vs. BG16; one-tailed test, $p=0.007$ ). Similarly, the same contract can be made more popular by introducing a decoy that is strictly dominated (G20 vs. BG16; one-tailed test, $p=0.009$ ). We summarize this in the following finding:

Finding 5. Unlike BG7, BG16 has significant $A E$ and CE (G18 and G20), but only for the nearly equal base contract, not for the more unequal base contract.

One interpretation of this finding is that there is more 'room' for the AE and CE to work in BG16 than in BG7. In BG16 it is therefore possible to raise agreements on the equal contract significantly via the $\mathrm{AE}$ and $\mathrm{CE}$. Note that, while there are significant $\mathrm{AEs}$ and $\mathrm{CEs}$ for the nearly equal base contract, it is still not possible to make the other, more unequal, base contract more attractive to the bargainers (cf games BG17 and G21).

\subsection{Decoy Properties}

In G19 the decoy offers equal payoffs, 14\% agree on it, and fewer (although not significantly, $p=$ 0.509 ) people agree on the target contract $B$ than in the absence of the decoy (BG16). The decoy is thus, if anything, 'counter-productive'. In G20, the base contracts are the same as in G19, but the decoy is now only nearly equal. When we compare G20 to BG16, we observe a significant increase in agreements on $B$ (one-sided test, $p=0.009$ ) and a decrease in agreements on $A$ (one-sided test, $p=0.051)$.

Finding 6. The AE for BG16 with a nearly equal payoff contract is significant only when the decoy offers nearly equal payoffs ( $c f$ G20), while it is insignificant when the payoffs of the decoy are perfectly equal (cf G19).

We think it is striking how such a little difference in the payoffs offered by the decoy makes a significant difference for the sign and magnitude of the AE. This suggest there is a significant behavioral difference between exactly and nearly equal payoffs not only for base contracts, but also for the decoy. ${ }^{25}$

We next consider if the strength of the CE depends on how extreme the decoy is. G8 and G9, and G10, G11 and G12, all based on BG7 with base contracts $(40,120),(60,60)$, differ in this

\footnotetext{
${ }^{25}$ We cannot with the current set of games say whether the significant AE in G20 is due to the target offering each player the same gain relative to the decoy, or whether it is because the decoy offers unequal earnings and hence does not compete in focality with the target. We investigate this in Experiment 2.
} 
respect. In none of the cases is there any CE, due to the overwhelming focality of contract $(60,60)$. We unfortunately do not in Experiment 1 have games that allow us to examine the effect of the extremeness of the decoy for other base games, but Experiment 2 explores this further.

We finally consider the role played by contracts that are strictly dominated by both base contracts. In G15 and G22 the contract $(30,30)$ is strictly dominated by both base contracts. In G22 this makes contract $(65,55)$ more frequently agreed on $(p=0.049)$, and $(40,120)$ less, although not significantly so $(p=0.120)$. Since $(30,30)$ is dominated by both base contracts, this is not a 'standard' AE. One conjecture for why adding $(30,30)$ makes agreements on $(65,55)$ more attractive is that $(30,30)$ is closer to $(65,55)$ than to $(40,120)$. In this sense $(30,30)$ serves as reference point for the bargainers. ${ }^{26} \mathrm{G} 15$ is similar to G22, but in the former game there is no significant effect of adding a dominated contract, since the focality of the equal payoff contract $(60,60)$ in BG7 is already so high.

Finding 7. Adding a contract $C$ that is dominated by both base contracts can raise agreement on one of them, even though $C$ is the only equal earnings contract.

\subsection{Agreement Times}

Although we are primarily interested in final bargaining outcomes, it is interesting to look also at the agreement times (cf Table 2). ${ }^{27}$ Note that there is no time pressure in our experiment other than the deadline. Provided that subjects agree on a contract before the deadline, they receive the points specified in the agreed contract (without any discounting); furthermore, even if they agree on a contract, they need to wait until the 120 seconds run out before starting the next round. In spite of this, there are clear differences in agreement times between the games. We present and discuss these differences in the online Appendix. Here, we only consider whether the addition of a third contract affects agreement times when we pool all games together.

Overall, we find that agreement times decrease by $12.76 \%(9.47 \%)$ in the games where we expect a CE (AE) compared to the base games. The drop is statistically significant ( $p=0.004$ for CE and $p=0.010$ for AE), and it holds even if we include the situations that ended in disagreement (by assigning them the maximum time of 120 seconds). In this latter case, agreement times decrease by $12.42 \%$ and $9.60 \%$, respectively ( $p=0.002$ and 0.007 ). Thus, the evidence suggests that adding a decoy speeds up the process of reaching an agreement, possibly by providing reasons to choose one base contract over the other (cf. Shafir et al., 1993).

\section{Experiment 2}

Experiment 1 identified significant AEs and CEs, but only under certain conditions. We conducted a second experiment to test whether the results from Experiment 1 can be replicated. We

\footnotetext{
${ }^{26}$ It is also the case that agreeing on $(65,55)$ gives players more similar gains than $(40,120)$, relative to $(30,30)$. Our data do not allow us to distinguish between the relative roles of closeness and equality of gains.

${ }^{27}$ There is a large experimental literature on bargaining with a 'deadline', see Karagözoğlu and Kocher (2019).
} 
also included new games to assess the robustness of the findings. The experimental design and procedures of Experiments 1 and 2 are exactly the same except for the list of the 22 games.

To determine the sample size for Experiment 2, we conducted an a priori power analysis. Assuming similar effect sizes to those observed in Experiment 1 (pooling all games re-included in Experiment 2), a type-I error rate of 0.05 for one-sided tests, and a power of 0.80 , we computed a sample size of 9 independent observations. In Experiment 2 we ran a total of 16 sessions (independent observations). So the sample size is comfortably above the required level.

\subsection{The Bargaining Games}

Table 3 and Figure 6 show the 22 games for which we collected data in Experiment 2. For ease of exposition, we can divide these games into four groups.

\begin{tabular}{c|ccc|c|c}
\hline \hline Game & \multicolumn{3}{|c|}{ Contracts } & Target & Hypothesized effect \\
\hline 1 & B & C & & - \\
2 & $(40,60)$ & $(60,40)$ & & - & Compromise \\
3 & $(40,60)$ & $(60,40)$ & $(80,20)$ & B & Attraction \\
4 & $(60,40)$ & $(50,30)$ & B & - \\
5 & $(80,120)$ & $(120,80)$ & & - & Compromise \\
6 & $(120,80)$ & $(160,40)$ & B & Attraction \\
7 & $(50,150)$ & $(120,80)$ & $(90,50)$ & B & - \\
8 & $(50,150)$ & $(150,50)$ & $(170,30)$ & B & Compromise \\
9 & $(50,150)$ & $(150,50)$ & $(130,30)$ & B & Attraction \\
\hline \hline 10 & $(40,120)$ & $(65,55)$ & & - & - \\
11 & $(40,120)$ & $(65,55)$ & $(120,40)$ & B & Compromise \\
12 & $(40,120)$ & $(65,55)$ & $(55,45)$ & B & Attraction \\
13 & $(60,140)$ & $(95,65)$ & & - & - \\
14 & $(60,140)$ & $(95,65)$ & $(140,60)$ & B & Compromise \\
15 & $(60,140)$ & $(95,65)$ & $(85,55)$ & B & Attraction \\
16 & $(60,140)$ & $(95,65)$ & $(80,40)$ & B & Attraction \\
\hline \hline 17 & $(40,120)$ & $(60,60)$ & & - & Compromise \\
\hline 18 & $(40,120)$ & $(60,60)$ & $(120,40)$ & B & Compromise \\
\hline 19 & $(40,120)$ & $(50,50)$ & & - & Compromise \\
\hline 20 & $(40,120)$ & $(50,50)$ & $(120,40)$ & B & Compromise \\
\hline \hline 21 & $(40,60)$ & $(60,40)$ & $(95,5)$ & B & Comprom \\
22 & $(40,120)$ & $(65,55)$ & $(155,5)$ & B & Comprom \\
\hline
\end{tabular}

Note: Base games $(1,4,7,10,13,17,19)$ are in gray.

Table 3: The bargaining games for Experiment 2.

Games 1-9: The first group consists of games with two symmetrically unequal base contracts. Games 1 (BG1), G2 and G3 are the same as the first three games of Experiment 1. By comparing behavior in these three games, we can verify whether previous results are reproducible with new 


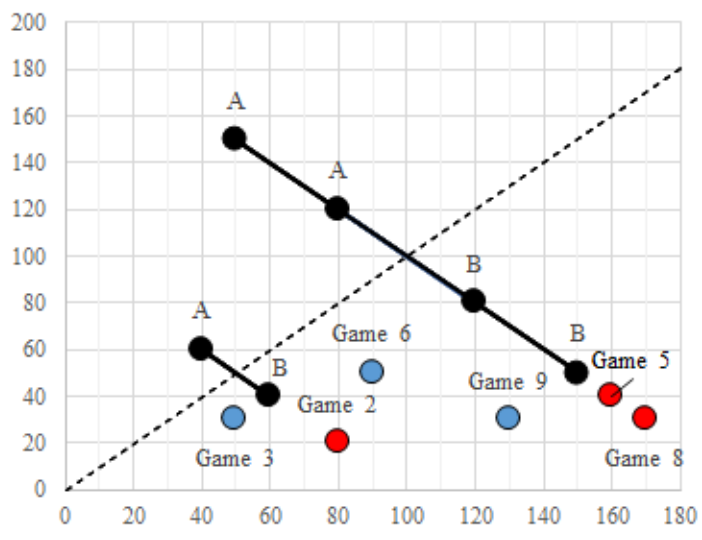

(a) Games 1-9.

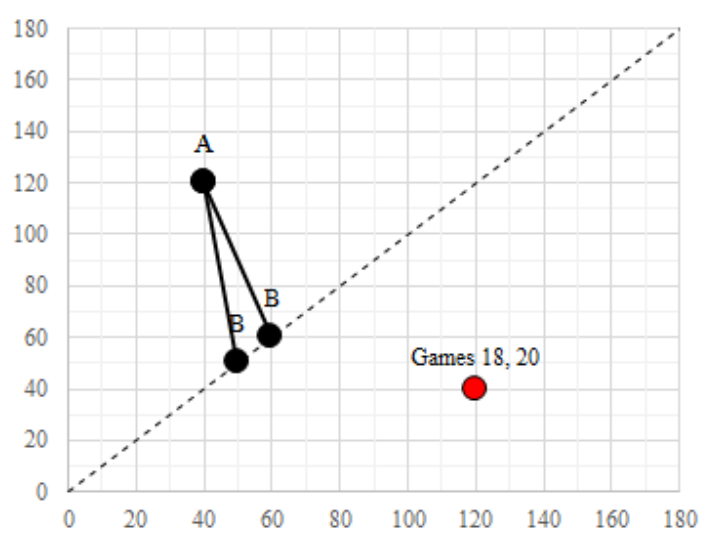

(c) Games 17-20.

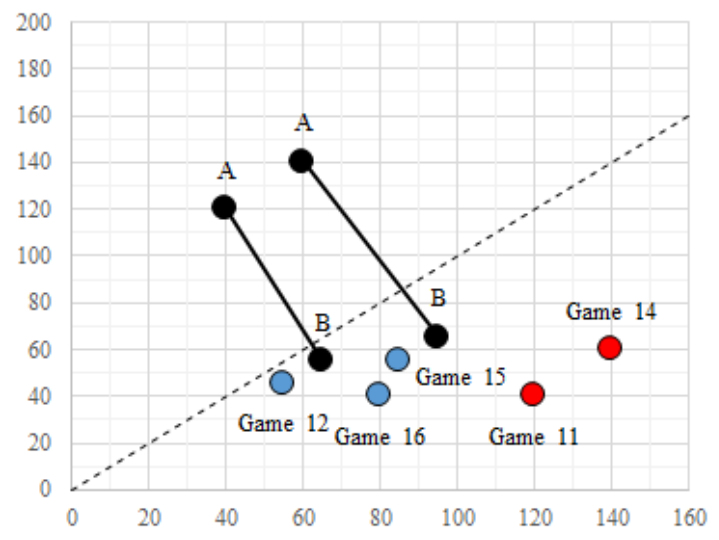

(b) Games 10-16.

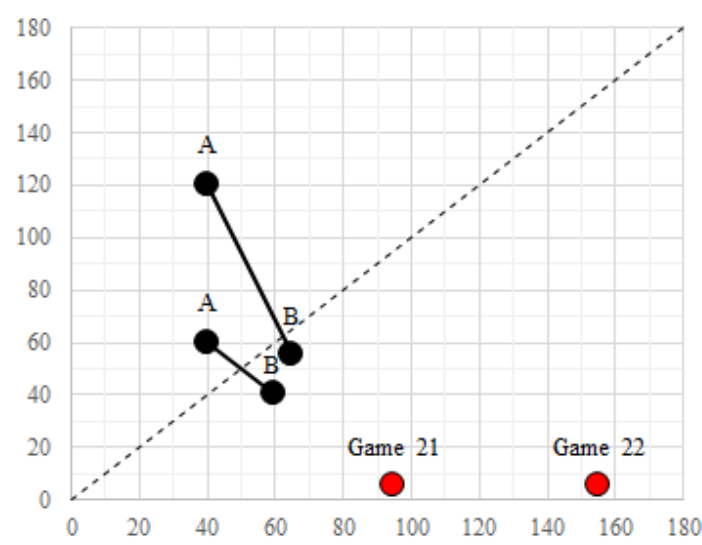

(d) Games 21-22.

Figure 6: Graphical illustration of the bargaining games in Experiment 2.

Note: The axes measure (in experimental points) how much each Person (1 and 2) gets for a given agreed contract. The base contracts $A$ and $B$ are in black. The decoy contract $C$ is in red (blue) when it is hypothesized to generate a $C E$ (AE).

participants. We also introduce additional games in order to test the robustness of our results. With respect to BG1, BG4 and BG7 vary the degree of inequality of the payoffs in the two base contracts. There is also some variation in the total payoffs at stake (BG4 and BG7 have larger total payoffs than BG1). If we consider the $\mathrm{CE}$, in $\mathrm{G} 2$ and $\mathrm{G} 5$ we introduce a decoy in such a way that the target $(B)$ is exactly in the middle: $B=(A+C) / 2$. In $\mathrm{G} 8$, the target is not exactly in the middle. Regarding the $\mathrm{AE}$, the target is always as unequal as the decoy, but the size of the gains changes (each player gains 10, 30 and 20 more in G3, G6 and G9, respectively, compared to the decoy).

Games 10-16: The second group consists of games where there is one unequal base contract (the target) competing with a second more unequal but total-earnings maximizing base contract. We include games that we already had in Experiment 1. These are games BG10, G11 and G12 where the less unequal contract is nearly equal. We also add new games (BG13, G14-G16) where the more equal base contact is far from being "nearly equal". In G14, we predict a CE, while in 
G15 and G16, an AE. In both G15 and G16, the decoy is constructed in such a way that it is at least as unequal as the target. This is to discourage subjects from agreeing on the decoy. In G15 (G16), players make equal (unequal) gains from the unequal decoy by agreeing on the target. In Experiment 1, we find a significant AE when the contracts are $(40,120),(65,55),(55,45)$. However, we cannot tell whether the AE occurs because the decoy is not equal or because the target offers the same gains relative to the decoy (see footnote 25). The comparison between G15 and G16 will shed light on this.

Games 17-20: In this group we consider games with an equal base contract which is the target of a CE. In Experiment 1, the equal base contract offered 60 experimental points to each player. We observed $90 \%$ agreements on that contract, leaving very little room for a CE to increase this proportion. In Experiment 2, we lower the payoffs of this contract in order to potentially create more "room" for a CE. First, we check whether we can replicate Experiment 1's results by including two games from the previous experiment. These correspond to BG17 and G18 in Experiment 2. We then consider two additional games (BG19 and G20) where we lower the payoffs of the equal base contract by 10 points.

Games 21-22: The last group of games are designed to study whether more extreme decoys are less effective in inducing a CE. Recall that, by extremeness, we mean how unequal the decoy is. More extreme decoys may be less effective in inducing a CE if players do not perceive them as credible candidates to be agreed upon and just ignore them. The results of Experiment 1 do not allow us to test for the extremeness of the decoy. In Experiment 1, we varied the extremeness of the decoy only for the base game $(40,120),(60,60)$ but we did not observe any CE at all in those games. In Experiment 2, we test for the extremeness of the decoy in games with two unequal base contracts. To economize on games, we construct the extreme decoys from existing base games. In particular, we consider G21 (to be compared with BG1) and G22 (to be compared to BG10). Decoys are more extreme in G21 and G22 than G2 and G11.

\section{Findings from Experiment 2}

The descriptive statistics for the 22 games are reported in Table $4 .{ }^{28}$ Figure 7 provides a visual representation of how agreements (and disagreements) vary between each game and the corresponding baseline game.

We first test whether there are $\mathrm{CE}$ and $\mathrm{AE}$ in all our games pooled together. The difference in agreement on the target contract between each game and the corresponding base game is significantly greater than zero, both in games where we hypothesize a CE (one-tailed test, $p=0.004$ ) and games where we hypothesize an $\mathrm{AE}$ (one-tailed test, $p<0.001$ ). This is in line with Finding 1 of Experiment 1, suggesting that both $\mathrm{CE}$ and $\mathrm{AE}$ are statistically relevant. We also find

\footnotetext{
${ }^{28}$ As for Experiment 1, we pooled the data across player labels (Person 1 or 2). Due to a connectivity problem between the server and the clients, we lost the data for a few bargaining interactions. Also, in half of the sessions, we dropped the data from G5 and G6 because we realized the two base contracts were identical instead of being symmetrical. A software fix resolved this problem for the remaining sessions.
} 


\begin{tabular}{|c|c|c|c|c|c|c|c|c|c|}
\hline \multirow{2}{*}{ Game } & \multirow{2}{*}{ Obs. } & \multicolumn{3}{|c|}{ Contracts } & \multirow{2}{*}{ Disagree } & \multirow{2}{*}{$\begin{array}{c}\text { Agree } \\
\text { on A }\end{array}$} & \multirow{2}{*}{$\begin{array}{c}\text { Agree } \\
\text { on B }\end{array}$} & \multirow{2}{*}{$\begin{array}{c}\text { Agree } \\
\text { on C }\end{array}$} & \multirow{2}{*}{$\begin{array}{c}\text { Time to } \\
\text { agree (in sec.) }\end{array}$} \\
\hline & & $\mathbf{A}$ & B & C & & & & & \\
\hline 1 & 127 & $(40,60)$ & $(60,40)$ & & $8.66 \%$ & $45.67 \%$ & $45.67 \%$ & $0 \%$ & 73.07 \\
\hline 2 & 128 & $(40,60)$ & $(60,40)$ & $(80,20)$ & $7.03 \%$ & $37.50 \%$ & $53.91 \%$ & $1.56 \%$ & 69.84 \\
\hline 3 & 127 & $(40,60)$ & $(60,40)$ & $(50,30)$ & $4.72 \%$ & $38.58 \%$ & $51.98 \%$ & $4.72 \%$ & 75.54 \\
\hline 4 & 128 & $(80,120)$ & $(120,80)$ & & $8.60 \%$ & $45.70 \%$ & $45.70 \%$ & $0 \%$ & 83.44 \\
\hline 5 & 64 & $(80,120)$ & $(120,80)$ & $(160,40)$ & $12.50 \%$ & $29.69 \%$ & $57.81 \%$ & $0 \%$ & 63.23 \\
\hline 6 & 64 & $(80,120)$ & $(120,80)$ & $(90,50)$ & $4.69 \%$ & $43.75 \%$ & $46.88 \%$ & $4.68 \%$ & 77.34 \\
\hline 7 & 128 & $(50,150)$ & $(150,50)$ & & $13.28 \%$ & $43.36 \%$ & $43.36 \%$ & $0 \%$ & 82.35 \\
\hline 8 & 127 & $(50,150)$ & $(150,50)$ & $(170,30)$ & $18.11 \%$ & $33.86 \%$ & $45.67 \%$ & $2.36 \%$ & 82.40 \\
\hline 9 & 127 & $(50,150)$ & $(150,50)$ & $(130,30)$ & $9.45 \%$ & $40.16 \%$ & $48.82 \%$ & $1.57 \%$ & 83.05 \\
\hline 10 & 127 & $(40,120)$ & $(65,55)$ & & $7.87 \%$ & $20.47 \%$ & $71.66 \%$ & $0 \%$ & 52.38 \\
\hline 11 & 128 & $(40,120)$ & $(65,55)$ & $(120,40)$ & $3.91 \%$ & $9.38 \%$ & $81.25 \%$ & $5.46 \%$ & 49.88 \\
\hline 12 & 127 & $(40,120)$ & $(65,55)$ & $(55,45)$ & $0.79 \%$ & $9.45 \%$ & $88.98 \%$ & $0.78 \%$ & 47.46 \\
\hline 13 & 128 & $(60,140)$ & $(95,65)$ & & $3.90 \%$ & $29.69 \%$ & $66.41 \%$ & $0 \%$ & 62.24 \\
\hline 14 & 128 & $(60,140)$ & $(95,65)$ & $(140,60)$ & $6.25 \%$ & $12.50 \%$ & $71.88 \%$ & $9.37 \%$ & 56.54 \\
\hline 15 & 128 & $(60,140)$ & $(95,65)$ & $(85,55)$ & $3.90 \%$ & $10.16 \%$ & $85.94 \%$ & $0 \%$ & 54.04 \\
\hline 16 & 128 & $(60,140)$ & $(95,65)$ & $(80,40)$ & $3.90 \%$ & $16.41 \%$ & $78.91 \%$ & $0.78 \%$ & 49.41 \\
\hline 17 & 128 & $(120,40)$ & $(60,60)$ & & $2.34 \%$ & $9.38 \%$ & $88.28 \%$ & $0 \%$ & 42.23 \\
\hline 18 & 126 & $(120,40)$ & $(60,60)$ & $(40,120)$ & $3.17 \%$ & $3.17 \%$ & $90.48 \%$ & $3.18 \%$ & 37.29 \\
\hline 19 & 128 & $(120,40)$ & $(50,50)$ & & $4.69 \%$ & $26.56 \%$ & $68.75 \%$ & $0 \%$ & 45.08 \\
\hline 20 & 128 & $(120,40)$ & $(50,50)$ & $(40,120)$ & $4.69 \%$ & $8.98 \%$ & $77.34 \%$ & $8.99 \%$ & 48.14 \\
\hline 21 & 128 & $(40,60)$ & $(60,40)$ & $(95,5)$ & $6.25 \%$ & $40.63 \%$ & $51.56 \%$ & $1.56 \%$ & 71.90 \\
\hline 22 & 128 & $(40,120)$ & $(65,55)$ & $(155,5)$ & $5.47 \%$ & $7.81 \%$ & $86.72 \%$ & $0 \%$ & 46.85 \\
\hline
\end{tabular}

Notes: For each game there are between 128 and 126 observations (number of pairs) except in Games 5 and 6 where we have only 64 observations (see footnote 28). Base games are shaded in grey. The contract labels $A, B$, and $C$ were not used in the experiment.

Table 4: Aggregate bargaining outcomes (Experiment 2).

heterogeneity in these effects across games. Regarding the CE, we find that the target contract is agreed on more frequently in G2 vs. BG1 (one-tailed test, $p=0.036$ ), G5 vs. BG4 (one-tailed test, $p=0.080$ ), ${ }^{29} \mathrm{G} 11$ vs. BG10 (one-tailed test, $p=0.012$ ), G20 vs. BG19 (one-tailed test, $p=0.013$ ), G21 vs. BG1 (one-tailed test, $p=0.072$ ), and G22 vs. BG10 (one-tailed test, $p=0.002$ ). Regarding the $\mathrm{AE}$, we find that the target contract is agreed on more frequently in G9 vs. BG7 (one-tailed test, $p=0.062$ ), G12 vs. BG10 (one-tailed test, $p<0.001$ ), G15 vs. BG13 (one-tailed test, $p=0.004$ ), and G16 vs. BG13 (one-tailed test, $p=0.009$ ).

\subsection{Games with an Equal Payoff Contract}

In comparing G18 with BG17, we replicate what we found in Experiment 1: roughly $90 \%$ of the bargaining pairs agree on the equal payoff contract, and there is no significant CE (one-tailed test, $p=0.286$ ). In BG19 and G20, we lower the payoffs of the equal contract by 10 points in order to reduce its attractiveness and test whether a decoy would now make the target more agreed upon. In BG19, subjects still agree mostly on the equal contract $B$, but significantly less often than in BG17 ( $p=0.001$ ). This gives more "room" for the CE, and we now observe a significant increase in the agreements on $B$ when we compare G20 to BG19 (one-tailed test, $p=0.013$ ).

Finding 8. In a game with an equal payoff but not total payoff maximizing contract, there is a significant CE on that contract if total payoffs are low enough.

\footnotetext{
${ }^{29}$ The CE in G5 is visibly large but it is significant only at the $10 \%$ level. This is because we collected fewer observations in G5. See footnote 28.
} 


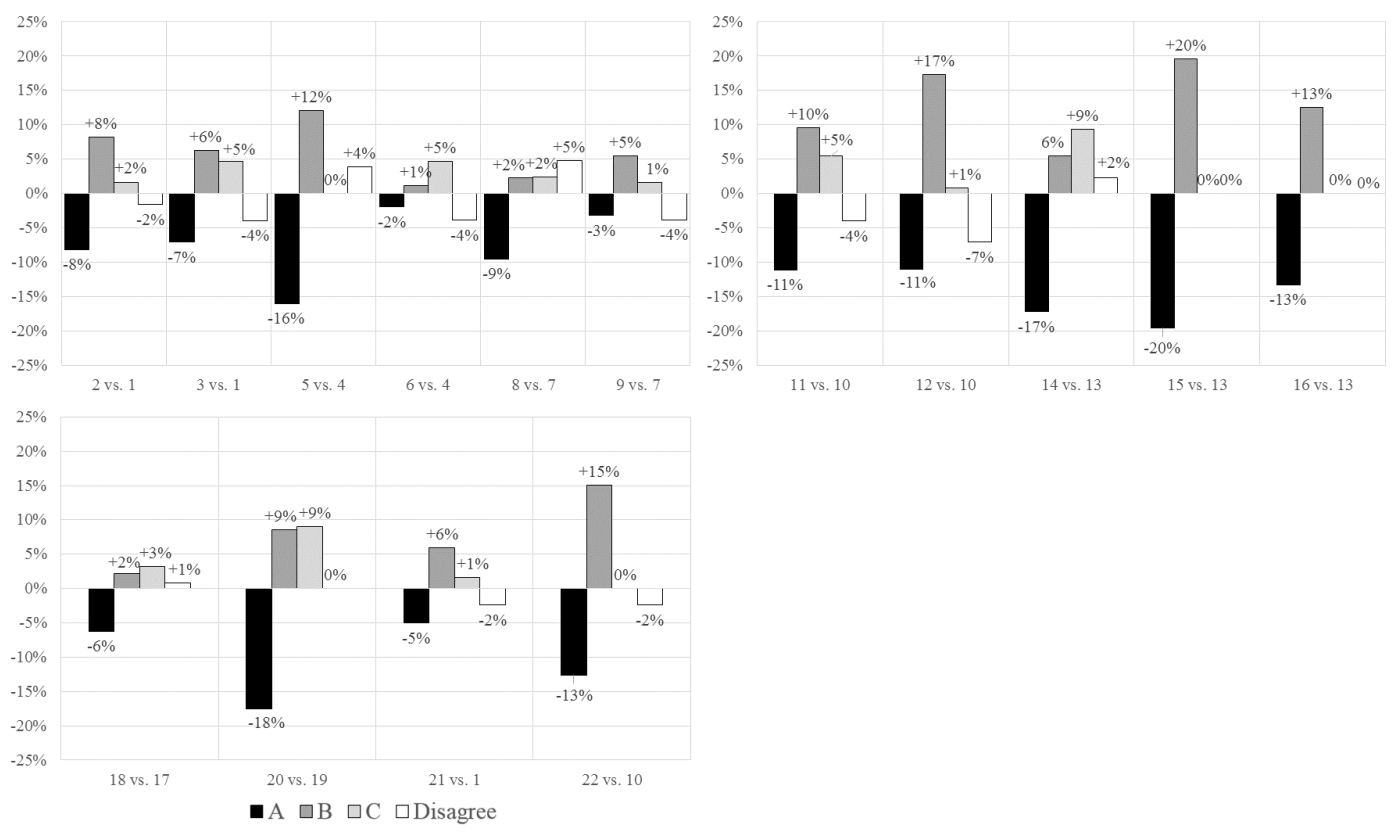

Note: the difference in the agreement rate on $C$ between a game and its corresponding base game is obtained by setting the agreement rate on $C$ equal to zero in the base game.

Figure 7: Changes in agreements on $A, B, C$ and disagreements for each game, compared to the base game

This result confirms previous evidence from Galeotti et al. (2019) showing that a significant CE exists in some (but not all) games with an equal but not total-earnings maximizing contract and this depends on the attractiveness of the equal payoff contract. ${ }^{30}$ Finding 8 suggests that we can expect the $\mathrm{CE}$ to be a quite generic phenomenon in unstructured bargaining with commonly known money payoffs: it remains significant even when one of the contracts offers equal but sufficiently low earnings.

\subsection{Games with Two Symmetrically Unequal Payoff Contracts}

If we consider jointly all games with two symmetrically unequal payoff base contracts (Games 1-9 and 21), we reject the null hypothesis that the average difference in agreement on the target contract between each game and the base game is equal to zero (one-tailed tests, $p=0.035$ for $\mathrm{CE}$ and 0.017 for $\mathrm{AE}$ ). This confirms that $\mathrm{AE}$ and $\mathrm{CE}$ are relevant in games with two symmetrically unequal payoff contracts. If we look at the games separately, we replicate Finding 3 from Experiment 1: subjects agree more on B in G2 than BG1 (one-tailed test, $p=0.036$ ), while the AE (G3 vs.

\footnotetext{
${ }^{30}$ Games 17-20 are also analyzed in Galeotti et al. (2019). They find a lower frequency of agreements on the equal contract in the base game, leaving more room for the CE to manifest itself. Similarly to our study, the CE effect is statistically significant only when the equal contract offers sufficiently low total payoffs compared with the unequal one (see footnote 39 in Galeotti et al. 2019).
} 
BG1) is not large enough to achieve statistical significance (one-tailed test, $p=0.121$ ). ${ }^{31}$

If we increase the inequality in the payoffs of the two base contracts (BG4 and BG7), we still find a significant CE in G5 (one-tailed test, $p=0.080$ ) ${ }^{32}$ but not in G8 (one-tailed test, $p=0.274$ ). ${ }^{33}$ A possible explanation is that the subjects whose preferred contract $(\mathrm{B})$ is made a compromise by the introduction of the decoy will bargain harder in favour of $\mathrm{B}$, while some of those who are in the weaker bargaining position (i.e. those who would get 50 from the compromise) are reluctant to accept the highly unequal contract B - even if it is a compromise and not the most unequal contract - and rather prefer to disagree. Note, however, that the decoy in G8 is not completely without effect. It reduces agreements on $A$ (one-tailed test, $p=0.029$ ) and slightly increases disagreement, though not significantly so $(p=0.188)$.

The inequality of the payoffs in the two base contracts does not appear to affect the AE. We observe an increase in the agreements on contract $B$ both in G6 and G9, but it is either not large enough to achieve statistical significance (BG4 vs. G6, one-tailed test, $p=0.285$ ) or it is only weakly significant (BG7 vs. G9, one-tailed test, $p=0.062$ ).

\subsection{Games with Two Asymmetrically Unequal Payoff Contracts}

Let us now consider games where there is one unequal base contract (the target) competing with a second more unequal but total-earnings maximizing base contract (G10-G16 and G22). If we pool together all games where we expect a $\mathrm{CE}$, we find that the average difference in agreement on the target contract between each game and the base game is positive and significant (one-tailed test, $p=0.002$ ). A similar result is obtained if we consider the games where we expect a AE (one-tailed test, $p<0.001)$.

If we look at the games separately, we fully replicate previous results from Experiment 1. In line with Finding 4, agreements on the nearly equal payoff contract in BG10 are significantly lower than agreements on the equal payoff contract in BG17 (the agreement rate on $B$ drops by almost $17 \%$ as in Experiment 1, $p=0.003)$. Also, the nearly equal payoffs contract $(65,55)$ can be made significantly more agreed on via a CE (G11 vs. BG10; one-tailed test, $p=0.012$ ) or an AE (G12 vs. BG10; one-tailed test, $p<0.001)$. This confirms Finding 5 from Experiment 1.

In the other games (BG13, G14-G16) the less unequal base contract is not anymore "nearly equal". In G14, we observe an increase in the frequency of agreements on contract B compared to BG13 (in line with a CE), but the effect is not large enough to achieve statistical significance with the sample size used (one-tailed test, $p=0.134$ ). In both G15 and G16, we observe a significant $\mathrm{AE}$ (one-tailed test, $p=0.004$ and 0.009). Recall that we find in Experiment 1 a significant AE when the decoy offers only nearly as opposed to exactly equal payoffs. However, we cannot tell

\footnotetext{
${ }^{31}$ If we pool the data of the two experiments together, the AE becomes (weakly) statistically significant (one-tailed test, $p=0.051$ ), suggesting that the AE is not totally absent but only less strong than the CE.

${ }^{32}$ See footnote 29 .

${ }^{33}$ Conflict is harsher in games BG7-G9 than in games BG1-G3 and BG4-G6. This is captured by the larger rate of disagreement and the fact that subjects took longer to agree in BG7-G9. On average, the disagreement rate is $13.61 \%$ in BG7-G9 against $6.81 \%$ in BG1-G3 ( $p=0.002)$ and $8.60 \%$ in BG4-G6 ( $p=0.031)$. Agreement times are, on average, higher in BG7-G9 than BG1-G3 (82.61 sec. vs. 72.83 sec., $p=0.006)$ and BG4-G6 (82.61 sec. vs. 77.01 sec., $p=0.109)$.
} 
whether this is because the decoy is not equal (and thus does not compete with the target in terms of focality) or because the target offers the same gain relative to the decoy. In G16 of Experiment 2 , the decoy offers more unequal gains relative to the target but, yet, we still find a significant AE. Hence, we can conclude that, in games with two asymmetrically unequal payoff contracts, AEs arise as long as the decoy is not equal and irrespectively of whether the target offers the same gain relative to the decoy.

\subsection{Games with Extreme Decoys}

We finally consider whether more extreme decoys are less effective in inducing a CE. We test that by comparing G21 and BG1, and G22 and BG10. If we pool the games together, we find that the average difference in agreement on the target contract between each game and the base game is positive and significant (one-tailed test, $p=0.003$ ). If we look at each game separately, we find a weakly significant CE in G21 (one-tailed test, $p=0.072$ ) and a significant CE in G22 (one-tailed test, $p=0.002)$. The increase in the frequency of agreements on the target is analogous if we compare G21 to G2 ( $p=0.714)$, and G22 to G11 ( $p=0.159)$, suggesting that more extreme decoys are as effective as more moderate decoys in inducing a CE.

\subsection{Agreement Times}

We can also, as for Experiment 1, consider the agreement times (cf Table 4). First of all, if we focus on the games included in both experiments, subjects were faster in reaching an agreement in Experiment 2 compared to Experiment 1 (on average, they were $14.02 \mathrm{sec}$. faster, $p<0.001$ ). The full list of games was not the same between the two experiments. Also, we conducted the two experiments in different years with different students. All this may explain this discrepancy in agreement times.

Despite this difference, most of the variation in agreement times across games observed in Experiment 2 is consistent with what we found in Experiment 1 (see the online Appendix for details). Also, if we pool the games together, we find that adding a decoy significantly reduces the time taken to agree by, on average, $7.76 \%$ for compromise situations ( $p=0.011)$ and $10.69 \%$ for attraction situations ( $p=0.023$ ). This result holds even if we include bargaining pairs that disagreed and set the agreement times for these pairs at 120 seconds. With the inclusion of the disagreeing pairs, creating a compromise still reduces agreement times by $6.64 \%(p=0.023)$, and an attraction does so by $12.22 \%(p=0.010)$. This confirms our results from Experiment 1 that adding a decoy contract speeds up the process of reaching an agreement.

\subsection{Econometric Analysis}

In the online Appendix, we examine all our data at once from both experiments using regression analysis to check the robustness of our results and test the simultaneous effects of the feasible 
agreements' characteristics on the $\mathrm{CE}$ and $\mathrm{AE}$. The analysis corroborates the main findings reported in the paper. In particular, we confirm that CEs increase agreements on the target if none of the base contracts offers equal payoffs, while they disappear if the non-targeted base contract gives equal payoffs or if the target contract is the most unequal. Similar patterns are detected for AEs. We also confirm that CE is less likely to manifest itself in highly conflictual games where the base contracts are symmetric and highly unequal. If the target contract offers equal payoffs, it is possible to observe a CE but only if the payoffs of the equal contract are low enough. If the payoffs are larger, the contract is almost universally chosen and there is not enough room for a CE to manifest itself. Finally, more extreme decoys are equally effective in inducing CEs. Interestingly, in all regressions reported in the online Appendix, AEs appear to be as relevant as CEs.

\section{Discussion}

In this section we discuss how changing some features of the design might impact the results.

First, the players' monetary payoffs are commonly known. This may not be a good assumption for many real bargaining settings. Each bargainer may know only his or her own payoffs from agreeing on a contract, see for example Roth and Murnighan (1982). One conjecture is that such private payoff information makes the $\mathrm{AE}$ and $\mathrm{CE}$ stronger since payoff focality, which is based on commonly known money payoffs, is muted. On the other hand, this kind of private payoff information also means that it is no longer from the outset common knowledge if a contract is a compromise or dominates other contracts. Thus it is a priori unclear what the strength of the AE and CE are in such a framework.

Second, we used an unstructured bargaining protocol, where players are free to make as many offers and counteroffers as they like. This is a feature of many real-world bargaining situations (for a recent review on unstructured bargaining experiments, see Karagözoğlu, 2019). In such an environment players have ample opportunities to coordinate on an agreement. It seems relevant to also study the $\mathrm{AE}$ and $\mathrm{CE}$ in an environment where the players need to coordinate quickly (modeled, in an extreme way, as a one-shot game).

Finally, we assumed there was a small number of feasible contracts. A question for future research is whether context effects of the type studied in this paper (bargainers choosing an intermediate alternative, or an alternative that dominates others) exist in settings with a much larger set of possible contracts.

\section{Conclusion}

The Attraction and Compromise Effects (AE and $\mathrm{CE}$ ) are celebrated findings from individual decision making. This paper reports the findings from what we believe is the first study of the role the $\mathrm{AE}$ and $\mathrm{CE}$ play in bargaining situations. In our bargaining environment, players negotiate over a set of feasible contracts, where a contract specifies an amount of money to each player. We define 
the $\mathrm{CE}$ as a situation where making a contract a compromise makes it more likely to be agreed on. The AE happens when a contract becomes more likely to be agreed on when it dominates another contract. These effects should not be observed according to the axiom of independence of irrelevant alternatives, or the Nash Bargaining Solution.

We systematically vary the money payoffs offered by the feasible contracts, and for each payoff condition we study the relevance of the AE and CE. Our experiments provide us with an impression of and prediction for when we should expect these effects to significantly matter in bargaining.

We observe that the $\mathrm{CE}$ is significant across a wide variety of payoff conditions, and varies with these in a quite intuitive way. The CE ceases to be significant if it targets the more unequal contract, if one of the contracts offers exactly equal and sufficiently high payoffs, or if the conflict between contracts whose payoffs are diametrically opposed becomes very high. Taken as a whole, the property of being a compromise per se is an important one in bargaining.

The $\mathrm{AE}$ is less robust than the $\mathrm{CE}$. Like the $\mathrm{CE}$, when there is no equal payoff contract, we observe that the AE can make a contract more likely to be agreed on if this contract is the less unequal contract. On the other hand, if the contracts are diametrically opposed ('symmetrically unequal' around the 45 degree line), and hence equally payoff focal, then the AE is less effective in increasing agreements on the target contract compared to the $\mathrm{CE}$. These novel findings can serve as a first guide to when we should expect the $\mathrm{AE}$ and $\mathrm{CE}$ to be present in bargaining situations.

We believe our findings are of significant practical interest for managers in a variety of situations. These include labor-management negotiations, wage negotiations, mergers \& acquisitions agreements, hiring decisions, business-to-business and intra-organizational negotiations, just to mention a few. Our results suggest that managers and business executives should carefully consider the set of available alternatives when they enter into negotiations with, for example, a labor union, a business partner or the head of another department. They should keep in mind that any seemingly irrelevant alternative that is brought to the table, intentionally or not, by any party in the negotiation may not be as innocuous as it appears to be. It may in fact have a considerable effect on the bargaining outcome, especially when it makes one of the other alternatives a compromise or a dominant alternative.

As a concrete example, consider hiring decisions. It is common to draw a shortlist and then choose a candidate out of this reduced set, usually after all candidates are interviewed. The decision of who gets an offer can be influenced by bargaining between (some of the) members of the interview panel, or between the manager of a department and his or her own line manager. Managers may wish to add a candidate that is dominated by their preferred candidate in order to highlight the qualities of their preferred candidate, or add an "extreme" version of their preferred candidate (one that may be unacceptable to the other members of the organization) in order to make their own candidate appear a compromise. Our findings suggest that a decoy will be more effective when it favors a candidate that has broad appeal (analogous to the least unequal contract) rather than a candidate who polarizes opinion (analogous to the more unequal contract). 
The use of decoys may or may not be advantageous to the organization as a whole. A common HR guideline for interview panels is to evaluate candidate responses/performance against the selection criteria for the role, rather than compare the candidate to other candidates. This guideline may be intended to mitigate context effects such as the ones studied in this paper.

\section{References}

Amaldoss, W., J. R. Bettman, and J. W. Payne, 2008, Biased but efficient: an investigation of coordination facilitated by asymmetric dominance, Marketing Science 27, 903-921.

Andreoni, J. and J. Miller, 2002, Giving according to GARP: an experimental test of the consistency of preferences for altruism, Econometrica 70, 737-753.

Bardsley, N., J. Mehta, C. Starmer, and R. Sugden, 2010, Explaining focal points: cognitive hierarchy theory versus team reasoning, The Economic Journal 120, 40-79.

Bateman, I. J., A. Munro, and G. L. Poe, 2008, Decoy effects in choice experiments and contingent valuation: asymmetric dominance, Land Economics 84, 115-127.

Beauchamp, J. P., D. J. Benjamin, D. I. Laibson, and C. F. Chabris, 2019, Measuring and controlling for the compromise effect when estimating risk preference parameters, Experimental Economics 23, 1069-1099.

Bolton, G. E. and E. Karagözoğlu, 2016, On the influence of hard leverage in a soft leverage bargaining game: the importance of credible claims, Games and Economic Behavior 99, 164-179.

Bolton, G. E. and A. Ockenfels, 2000, ERC: a theory of equity, reciprocity and competition, The American Economic Review 90, 166-193.

Bordalo, P., N. Gennaioli, and A. Shleifer, 2013, Salience and consumer choice, Journal of Political Economy 121, 803-843.

Brams, S. J. and D. M. Kilgour, 2001, Fallback bargaining, Group Decision and Negotiation 10, 287-316.

Bushong, B., M. Rabin, and J. Schwartzstein, 2020, A model of relative thinking, Review of Eonomic Studies, forthcoming.

Castillo, G., 2020, The attraction effect and its explanations, Games and Economic Behavior 119, 123-147.

Charness, G., U. Gneezy, and B. Halladay, 2016, Experimental methods: pay one or pay all, Journal of Economic Behavior \& Organization 131, 141-150. 
Charness, G. and M. Rabin, 2002, Understanding social preferences with simple tests, Quarterly Journal of Economics 117, 817-869.

de Clippel, G. and K. Eliaz, 2012, Reason-based choice: a bargaining rationale for the attraction and compromise effects, Theoretical Economics 7, 125-162.

Colman, A. M., B. D. Pulford, and F. Bolger, 2007, Asymmetric dominance and phantom decoy effects in games, Organizational Behavior and Human Decision Processes 104, 193-206.

Conley, J. P. and S. Wilkie, 1996, An extension of the Nash bargaining solution to nonconvex problems, Games and Economic Behavior 13, 26-38.

Conley, J. P. and S. Wilkie, 2012, The ordinal egalitarian bargaining solution for finite choice sets, Social Choice and Welfare 38, 23-42.

Crawford, V. P., U. Gneezy, and Y. Rottenstreich, 2008, The power of focal points is limited: even minute payoff asymmetry may yield large coordination failures, American Economic Review 98, 1443-1558.

Cunningham, T., 2013, Comparisons and choice, unpublished manuscript, Stockholm University.

Doyle, J. R., D. J. O'Connor, G. M. Reynolds, and P. A. Bottomley, 1999, The robustness of the asymmetrically dominated effect: buying frames, phantom alternatives, and in-store purchases., Psychology \& Marketing 16, 225-243.

Faillo, M., A. Smerilli, and R. Sugden, 2017, Bounded best-response and collective-optimality reasoning in coordination games, Journal of Economic Behavior \& Organization 140, 317-335.

Fehr, E. and K. M. Schmidt, 1999, A theory of fairness, competition, and cooperation, Quarterly Journal of Economics 114, 817-868.

Fischbacher, U., 2007, z-Tree: Zurich toolbox for ready-made economic experiments, Experimental Economics 10, 171-178.

Frederick, S., L. Lee, and E. Baskin, 2014, The limits of attraction, Journal of Marketing Research $51,487-507$.

Galeotti, F., M. Montero, and A. Poulsen, 2019, Efficiency versus equality in bargaining, Journal of the European Economic Association 17, 1941-1970.

Greiner, B., 2015, Subject pool recruitment procedures: organizing experiments with ORSEE, Journal of the Economic Science Association 1, 114-125.

Güth, W., S. Huck, and W. Müller, 2001, The relevance of equal splits in ultimatum games, Games and Economic Behavior 37, 161-169. 
de Haan, T. and R. van Veldhuizen, 2015, Willpower depletion and framing effects, Journal of Economic Behavior \& Organization 117, 47-61.

Herne, K., 1997, Decoy alternatives in policy choices: asymmetric domination and compromise effects, European Journal of Political Economy 13, 575-589.

Herne, K., 1999, The effects of decoy gambles on individual choice, Experimental Economics 2, 31-40.

Highhouse, S., 1996, Context-dependent selection: the effects of decoy and phantom job candidates, Organizational Behavior and Human Decision Processes 65, 68-76.

Huber, J., J. W. Payne, and C. Puto, 1982, Adding asymmetrically dominated alternatives: violations of regularity and the similarity hypothesis, Journal of Consumer Research 9, 90-98.

Huber, J. and C. Puto, 1983, Market boundaries and product choice: illustrating attraction and substitution effects, Journal of Consumer Research 10, 31-44.

Isoni, A., A. Poulsen, R. Sugden, and K. Tsutsui, 2013, Focal points in tacit bargaining situations: experimental evidence, The European Economic Review 59, 167-188.

Isoni, A., A. Poulsen, R. Sugden, and K. Tsutsui, 2014, Efficiency, equality, and labels: experimental evidence on focal points in explicit bargaining situations, The American Economic Review 104, 3256-3287.

Isoni, A., R. Sugden, and J. Zheng, 2020, The pizza night game: Conflict of interest and payoff inequality in tacit bargaining games with focal points, European Economic Review 127, 103428.

Kalai, E. and M. Smorodinsky, 1975, Other solutions to Nash's bargaining problem, Econometrica $43,513-518$.

Karagözoğlu, E., 2019, On "going unstructured" in bargaining experiments, in: J.-F. Laslier, H. Moulin, M. R. Sanver, and W. S. Zwicker, eds., The Future of Economic Design (Springer), 295-304.

Karagözoğlu, E. and M. G. Kocher, 2019, Bargaining under time pressure from deadlines, Experimental Economics 22, 419-440.

Kelman, M., Y. Rottenstreich, and A. Tversky, 1996, Context-dependence in legal decision making, The Journal of Legal Studies 25, 287-318.

Kıbris, Ö. and M. R. Sertel, 2007, Bargaining over a finite set of alternatives, Social Choice and Welfare 28, 421-437.

Kivetz, R., O. Netzer, and V. Srinivasan, 2004, Alternative models for capturing the compromise effect, Journal of Marketing Research 41, 237-257. 
Kőszegi, B. and A. Szeidl, 2013, A model of focusing in economic choice, The Quarterly Journal of Economics 128, 53-104.

Kuncel, N. R. and J. A. Dahlke, 2020, Decoy effects improve diversity hiring, Personnel Assessment and Decisions 6, 31-37.

Lichters, M., P. Bengart, M. Sarstedt, and B. Vogt, 2017, What really matters in attraction effect research: when choices have economic consequences, Marketing Letters , 1-12.

Lichters, M., H. Müller, M. Sarstedt, and B. Vogt, 2016, How durable are compromise effects?, Journal of Business Research 69, 4056-4064.

Lichters, M., M. Sarstedt, and B. Vogt, 2015, On the practical relevance of the attraction effect: a cautionary note and guidelines for context effect experiments, AMS Review 5, 1-19.

Luce, R. D., 1977, The choice axiom after twenty years, Journal of Mathematical Psychology 15, 215-233.

Luhan, W. J., A. U. Poulsen, and M. W. Roos, 2017, Real-time tacit bargaining, payoff focality, and coordination complexity: Experimental evidence, Games and Economic Behavior 102, 687 - 699.

Mariotti, M., 1998, Nash bargaining theory when the number of alternatives can be finite, Social Choice and Welfare 15, 413-421.

Masatlioglu, Y. and N. Uler, 2013, Understanding the reference effect, Games and Economic Behavior 82, 403-423.

Mehta, J., C. Starmer, and R. Sugden, 1994, The nature of salience: an experimental investigation of pure coordination games, The American Economic Review 84, 658-673.

Milberg, S. J., M. Silva, P. Celedon, and F. Sinn, 2014, Synthesis of attraction effect research: Practical market implications?, European Journal of Marketing .

Munro, A. and D. Popov, 2013, A portmanteau experiment on the relevance of individual decision anomalies for households, Experimental Economics 16, 335-348.

Myerson, R. B., 1989, Credible negotiation statements and coherent plans, Journal of Economic Theory 48, 264-303.

Nagahisa, R.-i. and M. Tanaka, 2002, An axiomatization of the Kalai-Smorodinsky solution when the feasible sets can be finite, Social Choice and Welfare 19, 751-761.

Nash, J., 1950, The bargaining problem, Econometrica 18, 155-162.

Noguchi, T. and N. Stewart, 2014, In the attraction, compromise, and similarity effects, alternatives are repeatedly compared in pairs on single dimensions, Cognition 132, 44-56. 
Ok, E. A., P. Ortoleva, and G. Riella, 2015, Revealed (p)reference theory, The American Economic Review 105, 299-321.

Pan, Y., S. O'Curry, and R. Pitts, 1995, The attraction effect and political choice in two elections, Journal of Consumer Psychology 4, 85-101.

Pinger, P., I. Ruhmer-Krell, and H. Schumacher, 2016, The compromise effect in action: lessons from a restaurant's menu, Journal of Economic Behavior \& Organization 128, 14-34.

Poterack, A., 2015, The compromise and attraction effects through frame preferences, Tech. rep., Mimeo, Boston University.

Roth, A. E., 1985, Toward a focal-point theory of bargaining, in: A. E. Roth, ed., Game-theoretic models of bargaining (Cambridge University Press), 259-268.

Roth, A. E. and J. K. Murnighan, 1982, The role of information in bargaining: an experimental study, Econometrica 50, 1123-1142.

Schelling, T. C., 1960, The strategy of conflict (Harvard).

Sedikides, C., D. Ariely, and N. Olsen, 1999, Contextual and procedural determinants of partner selection: of asymmetric dominance and prominence, Social Cognition 17, 118.

Shafir, E., I. Simonson, and A. Tversky, 1993, Reason-based choice, Cognition 49, 11-36.

Simonson, I., 1989, Choice based on reasons: the case of attraction and compromise effects, Journal of Consumer Research 16, 158-174.

Simonson, I. and A. Tversky, 1992, Choice in context: tradeoff contrast and extremeness aversion, Journal of Marketing Research 29, 281.

Slaughter, J. E., J. Bagger, and A. Li, 2006, Context effects on group-based employee selection decisions, Organizational Behavior and Human Decision Processes 100, 47-59.

Thomson, W., 1994, Cooperative models of bargaining, in: R. J. Aumann and S. Hart, eds., Handbook of Game Theory with Economic Applications, vol. 2 of Handbook of Game Theory with Economic Applications, chap. 35 (Elsevier), 1237-1284.

Trueblood, J. S., S. D. Brown, A. Heathcote, and J. R. Busemeyer, 2013, Not just for consumers: context effects are fundamental to decision making, Psychological Science 24, 901-908.

Tserenjigmid, G., 2019, Choosing with the worst in mind: a reference-dependent model, Journal of Economic Behavior \& Organization 157, 631-652.

Tversky, A. and I. Simonson, 1993, Context-dependent preferences, Management Science 39, 1179_ 1189. 
Wedell, D. H., 1991, Distinguishing among models of contextually induced preference reversals., Journal of Experimental Psychology: Learning, Memory, and Cognition 17, 767.

Yang, S. and M. Lynn, 2014, More evidence challenging the robustness and usefulness of the attraction effect, Journal of Marketing Research 51, 508-513. 